Revue d'histoire de l'Amérique française

REVUE D.HISTOIRE DE L'AMÉRIQUE FRANÇAISE

\title{
Un Canadien éminent
}

\section{Raphaël Bellemare (1821-1906) (suite et fin)}

\section{Marie-Claire Daveluy}

Volume 12, numéro 4, mars 1959

URI : https://id.erudit.org/iderudit/301935ar

DOI : https://doi.org/10.7202/301935ar

Aller au sommaire du numéro

Éditeur(s)

Institut d'histoire de l'Amérique française

ISSN

0035-2357 (imprimé)

1492-1383 (numérique)

Découvrir la revue

Citer cet article

Daveluy, M.-C. (1959). Un Canadien éminent : Raphaël Bellemare (1821-1906)

(suite et fin). Revue d'histoire de l'Amérique française, 12(4), 535-576.

https://doi.org/10.7202/301935ar d'utilisation que vous pouvez consulter en ligne.

https://apropos.erudit.org/fr/usagers/politique-dutilisation/ 


\section{UN CANADIEN ÉMINENT}

\section{RAPHAËL BELLEMARE (1821-1906) *}

membre fondateur de la Société historique de Montréal

(suite et fin)

1863. - PRÉfet de la CONGRÉgation DE MARIE IMMACULÉE A L'ÉGLISE SAINT-JACQUES, ÉTABLIE EN 1862, SOUS LA DIRECTION DE L'ABBÉ AUGUSTE CAMPION, P.S.S.

Voici une société qui engageait $M$. Bellemare à beaucoup de zèle en sa qualité de préfet. Elle lui créait aussi de nouvelles responsabilités. Heureusement, comme assistant, il comptait M. Olivier Berthelet, «ce logeur attitré de la plupart de nos communautés religieuses », a-t-on pu l'appeler, et Louis Giard; comme trésorier, M. Alfred Larocque dont la fortune favorisait la générosité naturelle et une foi très vive. Un an après sa fondation, cette société paroissiale se composait de 97 membres. Rien de plus édifiant sans doute à l'époque que de voir se glisser hors de leurs demeures, chaque dimanche, à $6 \mathrm{~h} .15 \mathrm{~m}$. du matin en été, à $6 \mathrm{~h}$. $30 \mathrm{~m}$. l'hiver, autant de chrétiens convaincus, heureux de rendre hommage à l'Immaculée dont le dogme défini récemment par l'Église, venait d'être sanctionné de façon émouvante par les apparitions de Lourdes. La fête patronale de la Société se célébrait le 8 décembre.

1863. - MEMbre DE LA SOCiÉTÉ DE TEMPÉRANCE DU DIOCÈSE DE MONTRÉAL, ÉTABLIE LE 5 JANVIER 1841, PAR SA GRANDEUR MONSEIGNEUR L'ÉVÊQUE DE NANCY [MGR DE FORBIN-JANSON] ${ }^{63}$ ÉRIGÉE CANONIQUEMENT PAR MANDEMENT DE MGR IGNACE BOURGET, ÉvÊQUE DE MONTRÉAL, EN DATE DU 25 JANVIER 1842, ET APPROUVÉE PEU APRÈS PAR N.S. LE PAPE GRÉGOIRE XVI.

\footnotetext{
* Voir, pour début de cet article, notre Revue, vol. XII : 35-55, 335-357.
} 
Cette société avait pris en peu de temps beaucoup d'extension. Elle nécessitait, en 1852, la création d'un Conseil central à Montréal. M. Bellemare, bien avant cette date, dut faire partie de la Société en qualité de membre ordinaire, peut-être vers 1847 ou 1848. Je n'en ai trouvé aucune preuve. Force m'est donc de m'en tenir aux renseignements donnés par l'Annuaire de VilleMarie. Ce qui accréditerait ma supposition, ce sont les lettres de Charles Chiniquy, presque toutes datées de Longueuil, 1850, et dans lesquelles il adresse des communications à M. Bellemare, rédacteur de la Minerve, afin d'appuyer, par ses articles la campagne de tempérance entreprise par ce journal. Chiniquy, alors ardent apôtre du mouvement, mettait même de la véhémence dans de simples billets. Le 28 novembre 1850, il débute ainsi dans les quelques lignes écrites à M. Bellemare: "La Religion avant tout! Vous savez comment ce qu'elle a de plus sacré a été traité l'hiver dernier par l'Institut [canadien]. Je ne puis donner plus longtemps la main aux hommes qui soutiennent les principes de l'Institut, me disait un des premiers citoyens de Montréal. Ces gens-là veulent renverser l'Église et quand elle tombera nous serons tous écrasés sous ses ruines. » Et Chiniquy riposte à «ce premier citoyen de Montréal»: «Non, l'Église ne retombera pas, car la presse, malgré ses torts immenses, s'est souvent mise du côté de Dieu, pour soutenir son plus bel œuvre... La Minerve s'est toujours montrée chrétienne, continue Chiniquy, depuis que j'y souscris, et c'est ce qui me fait espérer qu'elle ne refusera [pas] une place à la communication que je vous adresse. D'ailleurs cette communication ne fait allusion qu'à des faits déjà connus, quoique pas assez observés du public.» Ce pauvre Chiniquy, encore un peu de temps, et c'est à lui qu'on ne voudra plus tendre la main.

L'Annuaire de Ville-Marie nous donne la liste suivante des officiers qui dirigeaient la Société de Tempérance, dans le diocèse de Montréal: président d'honneur, Mgr Bourget, évêque de Montréal; président, M. C.-A. Leblanc; vice-présidents : MM. Narcisse Valois et Romuald Trudeau; secrétaire, Patrick O'Leary; trésorier, Hubert Paré; conseillers, Raphaël Bellemare... [et plusieurs autres personnalités en vue de notre ville]. 
1866. - COMITÉ D'ORGANISATION DE LA FÊTE DU 24 'MAI 1866, PAR les Anciens dU SÉminaire de Nicolet. Président, le JUGE THOMAS-JEAN-JACQUES LORANGER; TRÉSORIER, L'ABBÉ J.-N. TRUdEL, CURÉ DE SAINT-ISIDORE DE LAPRAIRIE; SECRÉTAIRES, RAPHAËL BELLEMARE ET SÉVÈRE RIVARD.

Il faut lire dans l'Histoire du Collège-Séminaire de Nicolet, par Monseigneur Douville, le récit enthousiaste qu'il nous donne de cet événement. "La grande réunion des anciens élèves, en 1866, fait époque, écrit-il, dans l'histoire du Séminaire. Elle fut, croyons-nous, la première de ce genre dans notre pays. » Un peu plus loin, il narre avec entrain quelques détails: « Le jeudi matin, 24 mai, la Mouche-à-feu et le Castor, ayant à bord environ quatre cents passagers quittaient Trois-Rivières, [lieu où tous s'étaient donné rendez-vous la veille], pour se rendre à Nicolet vers $10 \mathrm{~h}$. La musique du Collège dirigée avec beaucoup de talent et de succès par M. Hardy-Chatillon, ${ }^{64}$ attendait sur le quai. Le débarquement se fit le plus joyeusement du monde et la procession se forma pour se diriger vers le Séminaire en passant par cette magnifique avenue que ne manquent jamais de remarquer tous ceux qui visitent cette localité. Parmi les visiteurs, on remarquait Leurs Grandeurs les évêques des TroisRivières [Mgr Cook, son premier évêque], de Montréal [Mgr Bourget], et de Tloa [Monseigneur Baillargeon, administrateur de l'archidiocèse de Québec]; les abbés Laflèche, ${ }^{65} 0$. Caron, Cazeau et Isaac Desaulniers, ${ }^{66}$ vicaires-généraux; les honorables juges Mondelet et Loranger; des politiques éminents, PierreJoseph-Olivier Chauveau, Antoine-Aimé Dorion, Jacques Bureau, MM. Proulx et Olivier; des députés au Parlement, MM. Gaudet, de Niverville, et Bellerose; Sévère Dumoulin, maire de TroisRivières, Valère Guillet, le Dr Beaubien, et un grand nombre d'autres... L'adresse préparée par Raphaël Bellemare, fut lue par le juge Mondelet. Il y est fait mention des dons offerts à cette occasion au Séminaire par les Anciens. «Nous avons pris des arrangements, déclarent-ils, pour faire ériger dans votre chapelle, un orgue destiné à perpétuer le souvenir des rapports harmonieux qui existent entre les élèves de Nicolet et leur attachement à cette institution. » 
Puis, les Anciens y ajoutèrent un Album [au coût de cent dollars], contenant leurs photographies; et enfin, offrirent un cadeau personnel au Supérieur, l'abbé Thomas Caron, vicaire général et professeur de théologie au Séminaire. Il reçut un magnifique exemplaire in- 4 en douze volumes, des Oeuvres complètes de saint Thomas d'Aquin. Rappelons ici que, l'année suivante, le Comité des Anciens voulut marquer par de nouveaux cadeaux, le premier anniversaire de la mémorable journée du 24 mai 1866. Parmi ces dons, on notait la fondation à perpétuité d'un prix annuel dit «prix du souvenir » ou «prix des Anciens, en argent (douze dollars) décerné à l'élève qui terminerait son cours complet avec le meilleur succès dans toutes les parties de l'enseignement ».

Mgr Douville ne pouvait manquer de souligner «l'activité extraordinaire, le zèle qui n'avait eu d'égal que le succès de la manifestation» des deux secrétaires, MM. Raphaël Bellemare et Sévère Rivard.

1867. - COMité CANAdIEN DES ZoUAVES PONTIFICAUX FORMÉ LE 26 DÉCEMBRE 1867, À MONTRÉAL, AFIN DE DIRIGER LE MOUVEMENT DE DÉFENSE EN CE PAYS, EN FAVEUR DES ÉTATS PONTIFICAUX ATTAQUÉS PAR LES HORDES GARIBALDIENINES; AUSSI, D'ORGANISER UN DÉTACHEMENT DE VOLONTAIRES. L'EXÉCUTIF COMPRENAIT: M. OLIVIER BERTHELET, PRÉSIDENT; ET COMME MEMBRES: MM. LOUIS BAUDRY, ALFRED LAROCQUE, C.-A. LEBlaNC, RAPHAËL BELleMARE, FraNÇOISXavier Trudel, SÉvère Rivard, E. DE Bellefeuille, EDMUND BERNARD ET JOSEPH ROYAL.

La besogne de ces dix Montréalais distingués ne fut pas facile. Durant trois ans, aucun d'entre eux cependant ne se récusa, malgré les travaux ardus à accomplir, et les obstacles à surmonter. Ne s'agissait-il pas «du choix des soldats qui s'offraient par centaines, et venaient de tous les coins du Canada, de Montréal à Paspébiac (Bonaventure), et d'Ottawa aux autres villes du pays. Il fallait également percevoir des sommes considérables, prendre des mesures pour diminuer autant que possible 
les frais du voyage, veiller à sauvegarder les intérêts de ces jeunes volontaires et donner aux familles des garanties que leurs enfants auraient une protection efficace. $\gg 67$

Ces dix « Justes », choisis entre tant d'autres, furent magnifiques à la tâche et reçurent de Sa Sainteté Pie IX, des témoignages éclatants de sa reconnaissance. Le président, M. Berthelet, se vit conférer le Cordon de Commandeur de l'Ordre de Pie IX; les autres membres accusèrent réception «d'une lettre pleine de bienveillance dans laquelle Sa Sainteté exprime au Comité canadien des zouaves pontificaux, sa reconnaissance et son admiration pour tout ce qu'il a fait. ${ }^{68}$

1871. - OEUVRE DE LA FABRIQUE DE LA PAROISSE DE MONTRÉAL (Notre-DaMe). Établie le 28 aOÛt 1657. Premiers MarGUILLERS NOMMÉS LE 21 NOVEMBRE 1657.

M. Bellemare devint marguiller de la paroisse Notre-Dame en 1871, et demeura, durant vingt ans, membre de son Conseil d'administration.

1876 (?). - UNIVERSitÉ LAVAL DE MONTRÉAL.

M. Bellemare fut un des gouverneurs des premiers Conseils d'administration.

1878. - LA BANQUe D'ÉPARgne DE LA Cité ET DU District DE MONTRÉAL, FONDÉE LE 25 MAI 1846 SOUS LE PATRONAGE DE Sa Grandeur Mgr IgNaCe Bourget, Évêque de Montréal. M. Bellemare DeVint, EN 1878, DirecteUR DE LA BANQUe, PUIS EN 1890, VICE-PRÉSIDENT.

$\mathrm{Au}$ sujet de cette institution bancaire, l'Annuaire de VilleMarie (p. 106, note 1) nous fournit les informations suivantes. «Le Président actuel, y lit-on, est M. Alfred Larocque [en 1863]. Le bureau est ouvert tous les jours ... Les dépôts de vingt centins y sont reçus. Le nombre des déposants, au 31 décembre 1863, étaient de 3,813 et le montant déposé de $\$ 981,563$. Cette banque a distribué en 1863, aux institutions de charité de cette ville, une somme de $\$ 8,760$; ; et depuis sa fondation, celle de $\$ 24,260$. 
En 1946, à l'occasion du centenaire de la Banque, les directeurs confièrent à l'un de leurs collègues, M. Taggart Smyth, P.D. Sc., le soin de publier l'histoire de l'Institution. L'impression de l'élégante brochure intitulée « The First Hundred Years 》 n'a pas encore vu paraître la version française que l'on en a préparée. ${ }^{69}$

Nous rappellerons deux passages de cette œuvre. L'un a trait à Mgr Bourget: «It was His Grace [Mgr] Ignace Bourget, second catholic bishop of Montreal, who first conceived the idea of establishing the Savings Bank... He did not himself take any part in the administration, was never a member of its governing board, and at that period the Bank was a mutual institution, all of its earnings devolving to its depositors, after providing for the clerical staff and the necessary reserves for purposes of safety. Thus, there were for His Lordship neither wordly glory nor monetary gain. $\gg{ }^{70}$ Plus loin, M. Smyth remarque, tout en faisant l'éloge du deuxième président général de la Banque, M. Alfred Larocque, dont il note les hautes préoccupations sociales et le sens intelligent de l'épargne: «It was possibly his influence which induced His Grace Mgr Bourget to sponsor the establishment of a bank for savings. ${ }^{71}$ Montréal comptait alors 48,000 habitants. La plupart des Montréalais en vue pratiquaient volontiers une sage économie, étant souvent les propres artisans de leur fortune. Ils se montraient par-dessus tout des chrétiens fraternels et secondaient en ce sens leur évêque, dont tant d'œuvres secourables pouvaient se réclamer.

Le deuxième passage que nous citerons concerne M. Bellemare et la Notice biographique qui lui est consacrée dans le petit livre du Centenaire. C'est en sa qualité de directeur de la Banque durant 28 ans (de 1878 à 1906) et de vice-président durant 16 ans (de 1890 à 1906) que l'on désire tracer le portrait de cet éminent homme de bien. "M. Bellemare, écrit M. Smyth, has talents and tastes which inclined him to social enterprises $\gg{ }^{\mathbf{7 2}}$

1878-1879. - ProcÈs INFORMATIF diocÉSAIN DE LA VÉNÉRABle Marguerite Bourgeoys. DÉCret DE la SACRÉe CongrégaTION DES RITES DU 7 DÉCEMBRE 1878, INTRODUISANT LA 
CAUSE ET DÉClaRANT L/A vÉNÉRABILITÉ DE LA MÈRE BouRGEOYS.

Quelle fut la participation, au juste, de M. Bellemare, aux travaux effectués afin d'obtenir «l'heureuse issue de la première partie du procès de béatification et de canonisation $\gg$ de la fondatrice de la Congrégation de Notre-Dame, à Montréal ? Nous ne saurions le dire de façon certaine, mais M. Bellemare avait conservé une lettre de la Supérieure générale de la Congrégation, datée du 22 janvier 1879, accompagnée d'une copie du décret et d'une lettre de Mgr Charles-Édouard Fabre, évêque de Montréal, datée du 15 janvier 1879, annonçant le mémorable événement à 《ses chères filles» de la Congrégation de Notre-Dame.

Voici la lettre de la Supérieure générale, la Révérende Mère Saint-Victor, qui demeure un témoignage de l'attention apportée à la cause de la Servante de Dieu par M. Bellemare.

A M. R. Bellemare, Ecr. Avocat

22 janvier 1879

Monsieur,

J'ai l'honneur de vous offrir une copie du Décret que nous venons de recevoir de Rome. Veuillez l'accepter comme un bien faible tribut de la bien vive et respectueuse reconnaissance de notre Communauté, pour le concours généreux que vous avez bien voulu prêter à la cause de béatification et canonisation de notre Vénérable Fondatrice, dont l'introduction est actuellement le sujet d'une si grande et si douce joie dans notre famille religieuse. Je joins au Décret la lettre que Mgr de Montréal a bien voulu nous adresser à cette occasion.

Dans l'espérance que notre Vénérable Mère saura bien reconnaître ce que vous doit son Institut, en obtenant que le Ciel verse sur vous et sur tous ceux qui vous sont chers ses grâces les plus précieuses, je demeure, dans les sentiments d'une respectueuse estime et d'une haute considération,

Monsieur,

Votre très humble servante, Sœur Saint-Victor, sup ${ }^{\text {re }}$ gén $^{\text {le }}$ 
1901. - SOCIÉTÉ ROYALE DU CANADA. FONDÉE PAR LE MARQUIS DE LORNE, GOUVERNEUR GÉNÉRAL DU CANADA, LE 25 MAI 1882. M. BELLEMARE EN DEVINT MEMBRE EN 1901.

M. Bellemare fut certainement prié avant cette date d'entrer dans la Société Royale. Il y comptait de nombreux amis. Sans doute, estimait-il qu'il se révélait avant tout un chercheur laborieux, un «dénicheur» de vieux documents. Pourquoi prendre place parmi les littérateurs et les historiens de carrière. Mais ses travaux d'érudition qui allaient s'accumulant, surtout la thèse solide à laquelle il travaillait assidûment depuis 1900, afin d'établir scientifiquement les Bases de l'histoire d'Yamachiche, le désignaient de plus en plus fortement à l'attention des membres. Et voici qu'une place laissée vacante à la Société, le $\mathbf{1 5}$ mai 1901, au décès de l'abbé Verreau, président depuis 44 ans de la Société historique de Montréal, dont M. Bellemare demeurait le secrétaire, depuis 42 ans, ne fit que rendre plus opportune, plus désirable, la présence d'un érudit de même bon aloi que le regretté disparu. De nouvelles démarches furent tentées auprès de M. Bellemare qui accepta cette fois. Son acquiescement, n'en doutons pas, quoique souriant, voila mal un peu de mélancolie. Cet honneur lui advenait alors que ses 80 ans rendraient de courte durée l'assistance annuelle aux séances de la Société. Toutefois, dès l'année suivante, il eut le vif plaisir de voir monter à la présidence de la Section française, le fils aîné de son cousin et ami disparu, Antoine Gérin-Lajoie. Léon Gérin, économiste et sociologue de talent, lui écrivait peu après cette accession à la présidence, la charmante lettre que voici :

Ottawa, 24 mars 1902

Monsieur Raphaël Bellemare,

Montréal,

Cher M. Bellemare,

Vous avez été bien bon de prendre la peine de m'écrire au sujet [de mon travail] Notre Mouvement intellectuel, et de m'écrire dans une veine si 
aimable. Par un enchaînement de circonstances plus ou moins fortuites, je me suis trouvé un jour président de la Section française de la Société Royale, et il m'a semblé que le moins que je pouvais faire pour reconnaître le grand honneur que, par nonchalance ou indifférence, on avait laissé choir sur ma tête, c'était de me conformer au règlement et de faire la revue des matières relevant de la Section. A quoi bon, en effet, une Société qui ne fait pas œuvre collective, où chaque membre travaille dans l'isolement sans s'occuper du voisin, sans s'intéresser au mouvement général ? La Section française, il me semble, doit chercher à se tenir au courant du mouvement intellectuel du Canada français, chercher même, dans une certaine mesure, à diriger ce mouvement. Aussi, je me propose de faire cette année le même travail pour 1901, et je ne vois pas pourquoi, tous les ans, ou tous les deux ans, l'on ne ferait pas la revue des travaux publiés récemment par nos compatriotes. Un travail de cette nature, surtout s'il était fait en collaboration par plusieurs membres de la Section, aurait un intérêt réel.

Il m'a fait doublement plaisir de pouvoir mentionner votre nom et votre ouvrage, d'abord à cause du mérite intrinsèque de celui-ci, puis à cause de l'affection que mon père vous portait et que vous avez reportée sur nous. Je souhaite que Dieu vous conserve encore de longues années cette vigueur que vous mettez si bien à profit pour l'avantage du pays.

Croyez-moi, cher M. Bellemare,

Votre petit cousin bien affectueux

Léon Gérin

1900-1903. - COMITÉ POUR LA COMMÉmoRATION DU $2^{\text {e CENTE- }}$ NAIRE D'YAMACHICHE (1703-1903). ERECTION D'UN MONUIMENT À SAINTE ANINE, PATRONNE D'YAMAChICHE. M. BeLLEMARE EN FUT L'ANIMATEUR PRINCIPAL.

Peut-être est-ce trop peu dire que de citer M. Bellemare comme ayant été le grand animateur de cette fête magnifique 
du souvenir. Cet octogénaire distingué fut l'inspirateur, l'âme même de la manifestation. Depuis longtemps, il songeait à cette évocation des braves pionniers du sol d'Yamachiche, si bien représentés par les trois premiers colons d'Yamachiche: Étienne Gélinas, Jean-Baptiste Gélinas dit Bellemare, (trisaïeul de M. Bellemare), et Pierre Gélinas dit Lacourse. Le monument s'élevait sur la terre de l'un des trois premiers défricheurs, JeanBaptiste Gélinas dit Bellemare, dont les descendants de 1703 à 1903, de père en fils, en avaient possédé sans interruption la propriété. M. Bellemare fut vraiment d'une générosité princière en cette occasion. Il s'occupa de tout, même des plus minimes détails. Nous en avons là-dessus son propre témoignage, car chose curieuse, il dut défendre la légitimité et le désintéressement de son geste..$^{73}$ Il le fit, du reste, avec sa dignité habituelle et une émotion dont on reste frappé aujourd'hui encore.

M. Omer Héroux, fils lui-même du «vieux Machiche», a souligné dans une admirable préface qui ouvre le quatrième tome des Vieilles familles d'Yamachiche, par F.-L. Desaulniers, tout ce qu'un tel monument commémoratif pouvait éveiller dans l'esprit et le cœur des assistants. Il voulait dire: "une plus nette conscience de la grandeur et de la sainteté de l'idée de patrie, comme aussi de la noblesse de notre origine et des obligations qu'elle comporte. Nous aimerons plus et mieux notre petite patrie, continue-t-il, le coin de terre béni, aux horizons familiers, où chaque vallon, chaque paysage fait vibrer nos fibres les plus intimes; nous aimerons plus et mieux notre grande patrie, le cher et splendide pays où, nous en avons la fière ambition et le suprême espoir, notre race renouvellera son glorieux passé et refera les gestes de Dieu. 》

M. Bellemare, le vieil homme d'œuvres aguerri, infatigable, se sentit sans doute profondément heureux en lisant ces lignes frémissantes. Sa dernière initiative, le dernier groupement qu'il avait lui-même organisé cette fois, avait été compris et noblement jugé. Un jeune journaliste, par surcroît, un de ses «pays », au cœur vibrant et à la plume bien disante, lui en apportait le témoignage. 
LA CARRIÈRe INTELLECTUEL DE M. BELLEMARE - LE BIBLIOPHILE ET L'ÉRUDIT.

Les dispositions natives du troisième fils de Paul GélinasBellemare et de Marguerite Gélinas, firent présager assez tôt un avenir qui ne serait pas consacré aux travaux des champs, comme ses père et grand-père. Raphaël se révélait un enfant silencieux et méditatif, toujours en quête de savoir, attentif à toute conversation des Anciens, traitant de leur passé de labeurs et d'héroïque ténacité envers leurs traditions françaises et acadiennes. Car sa grand'mère paternelle, nous l'avons déjà rappelé, avait connu les angoisses et les misères de la déportation, en $\mathbf{1 7 5 5 .}$

Le curé d'Yamachiche, le bon, le saint M. Dumoulin, était ensuite apparu dans sa vie. Ce prêtre aux ailes de feu se penchait volontiers, à l'exemple du Maître, sur les petits enfants aux yeux attentifs. Il ne perdit point de vue le fils de Paul Bellemare et le conduisit jusqu'au seuil du collège de Nicolet.

Puis, ce furent les années heureuses à Nicolet, la connaissance des vieilles civilisations et de leur culture, l'éveil aux merveilles de la science; et, surtout, ce privilège d'une formation spirituelle qui ferait de sa longue existence un acquiescement continu, la consécration d'heures nombreuses aux œuvres de Dieu et du prochain.

Durant les vacances, voyons-le penché sur des livres, sur ses « livres de récompense ». Ils devenaient des amis, des intimes. Il les gardait à vue. Gare aux mains irrespectueuses pouvant les maculer. En lui naissait déjà le bibliophile, un fervent du livre sachant le priser d'abord pour sa valeur intrinsèque. M. Bellemare nous rappelle lui-même, du reste, dans son savant ouvrage sur Yamachiche (1904, p. 156), tout le cas qu'il faisait des premières œuvres de sa première et humble bibliothèque. N'allons pas croire cependant que les pages charmantes qu'il leur consacra aient eu un but de vaine gloriole. Non, cet octogénaire n'évoquera de tels souvenirs qu'en défendant noblement la langue française 
telle que la parlaient, en sa jeunesse, les Acadiens et les Canadiens français. Mais écoutons-le: "Nous voudrions convaincre, dit-il, les Anglais et les Américains, que de tout temps, ce n'était ni un patois, ni un jargon que les Acadiens et les Canadiens français parlaient sur le continent. Nous nous souvenons fort bien que, durant nos années de collège, dans les semaines de vacances, nous faisions des lectures en famille dans nos livres de prix. Quelquefois des voisins venaient les entendre et en parlaient à leurs amis avec éloges. Nous étions dans un rang habité par des Acadiens ou des fils d'Acadiens. Des vieillards, incapables de se déplacer pour cause de vieillesse ou d'infirmité, nous faisaient souvent savoir qu'ils seraient très heureux d'entendre ces lectures, dont on leur avait dit du bien. Pour leur être agréable nous nous rendions volontiers à leurs désirs...»

«La plupart de ces gens-là, continue M. Bellemare ne savaient pas lire, et cependant ils comprenaient et goûtaient même ces livres bien appréciés et populaires en France. Le Magasin pittoresque nous fournissait en partie ces bonnes lectures. Ce fait devrait suffire, nous semble-t-il, pour convaincre les plus incrédules des Américains que le langage des Acadiens et des Canadiens français n'était pas un patois puisque des illettrés comprenaient si bien cette littérature française de bon aloi ... Le Français lettré peut converser avec eux, aussi facilement sinon mieux qu'avec les paysans illettrés de plusieurs départements de la vieille France. Ce témoignage nous a souvent été rendu par des Français observateurs, notamment par M. Rameau, qui en avait, à dessein, fait l'épreuve durant son séjour en Canada comme en Acadie. 》

Mais ne quittons pas encore Nicolet et ses éminents professeurs. Car, c'est en cette institution que s'éveilla chez M. Bellemare, cette deuxième vocation qu'il a semblé aimer plus que toute autre, celle d'érudit en histoire canadienne.

Voici les faits. Un jour, des circonstances douloureuses, le décès du Supérieur, l'abbé Jean Raimbault, qui se dévouait à l'enseignement au Collège de Nicolet, depuis trente-cinq ans, de 1806 à 1841, amenèrent à cette maison, un nouveau directeur et préfet des études. Ce remplacant n'était nul autre que l'abbé 
Jean-Baptiste-Antoine Ferland. Plusieurs le connaissaient déjà, avaient désiré sa venue, vantant à l'envie sa science et sa bonté de cœur. Raphaël Bellemare avait alors vingt ans. Il fut impressionné à son tour, dès sa première rencontre avec ce prodigieux abbé Ferland. Tout en l'enseignement qu'il projetait, en ses méthodes de culture, en l'atmosphère qu'il créait déjà autour de lui, répondait aux meilleures aspirations de son esprit et à l'idée qu'il se faisait du prêtre éducateur. Il s'abandonna totalement à sa direction. Aussi bien, quelle préparation à la vie, sous quelque forme qu'elle se présenterait bientôt à lui, ne recevrait-il pas auprès de ce maître à la foi rayonnante, à la raison claire, au sens aigu des réalités de ce monde, dont il savait tirer de splendides leçons ! Quand il parlait d'histoire canadienne, sa ferveur et sa certitude se communiquaient aux élèves. De beaux documents, commentés devant tous, avec tous, venaient sans cesse appuyer les assertions du professeur. Lorsque Raphaël Bellemare, après deux années de théologie et de professorat, quitta, non sans regret, le CollègeSéminaire de Nicolet, il avouait que l'influence du docte M. Ferland s'exercerait encore longtemps sur lui. De fait, le maîtrehistorien, avec la cordialité affectueuse qui lui était si naturelle, échangea jusqu'à la fin de sa vie, avec plusieurs de ses élèves, une correspondance fort instructive. Ici encore, citons le témoignage de M. Bellemare, octogénaire. « Notre savant et estimable historien, M. l'abbé Jean-Baptiste-Antoine Ferland n'avait pas oublié son très humble élève et servant de messe au Collège de Nicolet; même au milieu de ses grands travaux, en poursuivant ses recherches historiques à Québec, il pensait à nous. S'il trouvait quelques faits pouvant nous intéresser personnellement, il daignait s'interrompre pour nous les transmettre. Nous lui devons, entre autres [une lettre] au sujet de notre aïeul [Étienne Gélinas]. 》 74

Que découle-t-il de ce tableau d'ensemble rétrospectif, de ce prélude en quelque sorte, aux vocations de bibliophile et d'érudit chères à $M$. Bellemare ? La certitude que celui-ci, durant sa jeunesse, fut vraiment un esprit chercheur, avide de science; un liseur aussi, un ami des livres, fussent-ils modestes d'apparence, 
mais riches de pensée et de savoir. Ne s'en faisait-il pas même l'apôtre, parfois, comme on vient de le voir ?

Mil huit cent cinquante-six fut une année que M. Bellemare se rappela toujours avec plaisir. Il comptait, en février, trentecinq ans d'âge. Il pouvait déjà dresser un bilan intéressant des labeurs intenses auxquels il s'était livré depuis son arrivée à Montréal, durant l'été de 1847. Résumons-les: cinq ans de journalisme ardu, semés de périls, ${ }^{75}$ initiation à la politique, celle de son pays surtout, en des temps difficiles, orageux, tragiques; contact fréquent avec des hommes célèbres, qui firent l'histoire, chez nous, à cette époque; puis, à la mort de Ludger Duvernay, le voici journaliste intermittent et étudiant en droit. Durant trois ans il fréquenta des juristes dont la postérité a recueilli les noms; enfin, admis au barreau de la province de Québec en 1855, il songea à ouvrir une étude légale. Il n'exerça jamais sa profession cependant, car c'est en 1855 que le Ministère, à Québec, lui offrit une situation assez lucrative, celle d'inspecteur du revenu provincial. Il accepta ce fonctionnariat qui lui assurait une aisance matérielle fort désirable pour le foyer qu'il s'était créé, il y avait déjà six ans. N'est-il pas permis de croire qu'il songeait aussi aux loisirs qui apparaîtraient davantage dans sa vie, grâce à cette stabilité financière ? Ses études historiques, jamais délaissées, toujours aimées, se poursuivraient avec plus d'assiduité et de facilité.

Tout convergea bientôt pour le favoriser en ces matières. C'est en 1856 certainement que M. Bellemare noua des relations avec l'érudit montréalais Jacques Viger, «l'historien consultant de son époque », comme le qualifiait un jour Mgr Camille Roy. Comment la première rencontre s'opérait-elle ? Le plus simplement du monde. Le 2 juin 1856, le Commandeur Viger était élu (pour la deuxième fois) président de la Société Jean-Baptiste de Montréal, dont Raphaël Bellemare était le secrétaire depuis huit ans. Président et secrétaire, dans toute société, ne doivent-ils pas marcher la main dans la main, s'ils ne désirent pas voir grincer les rouages de l'entreprise ? La différence d'âge ne nuit pas, bien au contraire. Le respect du plus jeune devient une sorte d'attitude diplomatique qui éloigne bien des heurts. En le cas 
présent le distant et silencieux M. Bellemare, si compréhensif pourtant, plut beaucoup à M. Viger, enclin à la critique et aux remarques parfois très acerbes. Que $\mathrm{M}$. Bellemare, apparemment, ne parût jamais impressionné par l'humeur ironique de son chef, humeur encore accrue par la maladie, durant les dernières années de sa vie, réjouissait fort M. Viger. Il s'attacha, peu à peu à ce jeune secrétaire. Il finit par désirer sa présence, et les remarques de son esprit réfléchi. Nous avons devant nous deux courts billets adressés à M. Bellemare par M. Viger qui le convoquait fort courtoisement chez lui. Voici la teneur de ces invitations:

\section{3 janvier [1857?]}

Que fait M. Bellemare, ce soir ? J'ai reçu une lettre de M. de L. ? que je n'ai pas encore ouverte... S'il [M. Bellemare] n'a rien de mieux à faire, qu'il vienne donc tôt ou tantôt s'ennuyer quelques instants

avec J. Viger

Le deuxième billet n'est pas daté et signé d'initiales seulement. Mais après lecture du texte, on peut y ajouter l'année 1857.

Me voilà, écrit $M$. Viger, encore détenu par ma jambe. M. Verreau m'a donné à entendre hier qu'il viendrait me voir tantôt. Venez si ça vous plaît \& convient.

Dimanche.

J.V.

M. Viger demeurait alors au No 24, rue Notre-Dame. M. Verreau, nommé principal de l'École Normale Jacques-Cartier, venait de s'installer dans les locaux de l'École, rue Saint-Claude, c'est-à-dire, dans les dépendances de l'ancien Hôtel du Gouvernement (le Château de Ramezay). Le vieux manoir du régime français était occupé par la Surintendance de l'Instruction publique que dirigeait Pierre-Joseph-Olivier Chauveau. Où demeurait M. Bellemare ? D'après l'adresse d'une lettre de l'époque, il logeait rue Sanguinet, près de la rue Sainte-Catherine. Les distances à parcourir n'étaient donc pas longues pour MM. Verreau et Bellemare. Ils se retrouvaient vite auprès du fauteuil du vieux 
Commandeur, souvent indisposé, durant les deux dernières années de sa vie.

On devine la satisfaction, la joie des deux visiteurs, de pénétrer dans un milieu où d'innombrables documents historiques avaient été réunis par les soins et aux frais de ce premier maire de Montréal, archiviste-né, familier avec la grande et petite histoire canadienne. De son côté, M. Viger n'avait peut-être jamais connu de disciples aussi bien préparés à l'étude des questions historiques que MM. Verreau et Bellemare, auxquels vinrent bientôt se joindre deux admirateurs de la personne et de l'œuvre amassée par M. Viger, dans ce qu'il appelait «Ma Saberdache rouge » (30 registres) et « Ma Saberdache bleue» (13 registres). Les derniers à accourir en ce cénacle historique étaient JosephUbald Baudry, greffier des Appels, ${ }^{76}$ une autorité en tout ce qui concernait l'organisation judiciaire du Canada, et Louis-François-Georges Baby, ${ }^{77}$ un tout jeune homme de 25 ans admis au Barreau depuis le 26 août 1857. On le voit, il y avait entre les disciples de M. Viger, septuagénaire, une variété d'âge allant de 48 à 25 ans.

Et l'on se mit à deviser avec ardeur sur nos annales. M. Viger avait réponse à tout. Les documents qu'il possédait émerveillaient ses compagnons. Constamment consultés ils n'épuisaient point la richesse de leur enseignement. Qui peut s'étonner que bientôt ait pris naissance, dans l'esprit du Commandeur, charmé de l'atmosphère créée par ces hommes d'études, le projet de fonder une Société d'histoire ? Il terminerait logiquement, heureusement ainsi, son existence tournée si volontiers vers les problèmes de l'érudition historique. A la fin de l'été 1857, ou au commencement de l'automne, la fondation de la Société historique de Montréal était chose faite, grâce à l'acquiescement unanime et enthousiaste de ce groupe de chercheurs. On est enclin à donner l'année 1858 comme date de fondation de cette société historique. On fait erreur. M. Viger, (décédé le 12 décembre 1858) fixe la fondation à l'année 1857 et l'organisation définitive à 1858. Le Notaire Huguet-Latour, le premier membre à s'inscrire dans la Société, donne lui aussi, dans son Annuaire de Ville-Marie, l'année 1857, comme date de fondation. Évidem- 
ment les fondateurs ne nous indiquant point le quantième ni le mois de l'événement, il nous est loisible d'en commémorer le souvenir durant les derniers et les premiers mois de 1857-1858.

Ce fut certainement un événement qui fit sensation et même du bruit à Montréal. L'on applaudit en beaucoup de milieux, grâce à la liste imposante des sociétaires divulguée un an après sa fondation. L'on savait surtout que l'honorable Louis-Hippolyte LaFontaine y avait donné une conférence, qu'il en était, comme l'on disait alors, le patron distingué. Mais les attaques surgirent aussi. Maximilien Bibeau exhala sa mauvaise humeur dans une brochure intitulée: La Société historique de Montréal. Portraits des Collitigans (Montréal, des Presses de Cérat et Bourguignon, [1859], 12 pages). Le cousin Antoine Gérin-Lajoie a bien jugé cette intervention de $\mathrm{M}$. Bibaud, dans une lettre à son ami, Raphaël Bellemare. Je m'empresse, ayant cette lettre sous la main, et sachant qu'elle est inédite, de la transcrire ici :

Toronto, 28 mars [1859]

Mon cher Bellemare,

Rolland m'a envoyé le petit pamphlet ${ }^{78}$ de Bibaud contre la Société historique. C'est bien le comble du ridicule. Je ne serais pas surpris de voir avant six mois ce prétentieux écrivain battre la campagne. Je vois que j'ai aussi un petit coup de patte dans l'avant-dernière page. Mais M. Bibaud se trompe; qu'il regarde bien droit en examinant mon tableau des membres [du Parlement], et il verra que M. Papineau n'est pas mis pour Huntingdon mais pour Kent. Mes écrits sont assez incorrects sans cela, et s'il fallait à mes erreurs ajouter celles de M. Bibaud, je resterais certainement éreinté du coup.

Quant à vous, illustres Collitigans, vous n'avez qu'à vous féliciter d'avoir été jugé dignes de la colère de ce Bonaparte intellectuel du Canada.

Je suppose que l'article sur les LeMoyne dans une des dernières Minerve va vous attirer une nouvelle bordée. Heureusement que le public et les vieux sont de votre côté.

Tout à toi,

A.G L. 
Le cousin Antoine coulait maintenant des jours heureux. En 1856, en avril, son ami Louis-Victor Sicotte, président de la Chambre d'Assemblée, réussissait à l'adjoindre au nouveau bibliothécaire, Alpheus Todd, chargeant tout particulièrement Antoine Gérin-Lajoie de la section française. En 1858, le 26 octobre, il épousait Joséphine Parent, fille aînée d'Étienne Parent, publiciste et fonctionnaire. Sa vie, cependant, demeurait nomade. Il se transportait, soit à Toronto, soit à Québec, selon les villes où le gouvernement se rendait pour siéger. Après la Confédération, en 1868 , il se fixera définitivement à Ottawa. La correspondance restait toujours active entre les cousins qui se rencontraient au moins une fois l'an. Il en avait été ainsi jusqu'à la mort d'Antoine, en 1882.

Revenons à M. Viger et à la Société historique de Montréal. Elle ne garda pas longtemps son président-fondateur. Le 12 décembre 1858, il s'éteignait, âgé de 71 ans. Le vice-président, Raphaël Bellemare, dut convoquer une assemblée dès janvier. Elle élit, à l'unanimité, comme président, l'abbé Hospice-Anthelme Verreau.

M. Bellemare appréciait beaucoup le successeur de M. Viger. Des goûts communs les rapprochaient et les caractères s'harmonisaient. Peu de temps suffit pour créer entre eux, outre des relations agréables, une sincère affection. On ne peut nier que la Providence comblait de nouveau les vœux de M. Bellemare. Le choix de ses maîtres et compagnons continuait d'être bienfaisant. Après l'abbé Ferland, après $M$. Viger, un troisième érudit, l'abbé Verreau, entrait dans sa vie. Bien qu'il n'eût que 30 ans, M. Verreau possédait une belle maturité d'esprit, beaucoup d'autorité, à laquelle son attitude réservée, d'une parfaite dignité, ajoutait encore. Ce principal de l'École normale Jacques-Cartier, cet éducateur-apôtre, avait voué un culte à l'histoire de son pays. Il apportait dans ses travaux un souci de la vérité qui le retenait auprès des seules sources originales interprétées avec sûreté, grâce à une science rigoureuse. M. Bellemare avait lui aussi les qualités et les méthodes du véritable historien et s'enchantait de plus en plus de ce compagnon de route. La Société historique ne pouvait que se ressentir de la vigilance de ces deux chefs. Sa réputation grandissait peu à peu. 
M. Victor Morin nous a donné une excellente étude ${ }^{79}$ sur la dernière initiative en histoire de $M$. Viger. Il nous a aussi fait connaître la liste des premières études présentées à la Société. D'après le Mémoire sur la Société, dressé en 1872 par le président, M. Verreau, et le secrétaire-adjoint, M. J.-A. Danis, il appert que sur les 28 travaux dont la Société se prévalait, 17 sont l'œuvre de MM. Verreau et Bellemare. Voici les sept études préparées par M. Bellemare :

Le Site des anciens cimetières de Montréal. Samuel de Champlain. - Le véritable nom du Cap de Chattes. - L'affaire du fort Nécessité et des otages Van Braam et Stobo. - Trois lettres autographes de M. David, missionnaire, à Monseigneur Bruté. - Notice historique sur la Saint-Jean-Baptiste. - La famille de Rocheblave. ${ }^{80}$

Nous savons que la publication des Mémoires de la Société - cinq volumes, de 1859 à 1872, - était l'objet d'un examen final par MM. Verreau et Bellemare, avant d'être livrés à l'imprimeur. Parfois aussi, tous deux y collaboraient, tantôt comme éditeur ou annotateur, tantôt comme auteur. La correspondance entre MM. Verreau et Bellemare se prolongeait chaque été, car le Principal de l'École Normale aimait à se reposer durant les vacances, dans la quiétude de l'Islet, son village natal. Une quinzaine de lettres que nous conservons avec soin, nous apportent la preuve de la sollicitude intellectuelle exercée par ces distingués sociétaires, sur le groupement qu'ils dirigeaient.

M. Victor Morin et quelques autres conférenciers et journalistes ont souvent entretenu les membres de la Société de l'œuvre capitale de M. Viger, «Ma Saberdache». Ils ont déploré que cette collection de documents eût été léguée par M. Verreau, à sa mort, non à la Société historique de Montréal, mais au Séminaire de Québec. Ils estimaient même que certaines clauses du testament de Mesdemoiselles Lennox, propriétaires des manuscrits et livres de M. Viger, mort ab intestat et déclaré insolvable après l'inventaire de ses biens, pouvaient être interprétés en faveur de la Société historique. Maintenant que toutes les pièces légales relativement à cette affaire assez compliquée sont mieux 
connues, l'on en juge autrement. Quoi qu'il en soit, car je ne puis entrer ici dans des détails hors de ma compétence, voici trois lettres de Mgr Thomas-Étienne Hamel, bibliothécaire à l'Université Laval de Québec, à la mort de l'abbé Verreau et qui les signait en qualité de «procureur de Dlle Justine Verreau, Exécutrice testamentaire » (ce sont ses propres expressions). Elles étaient adressées à M. Raphaël Bellemare, à Montréal. Ces missives pourraient être ajoutées aux autres pièces du dossier, en les accompagnant, naturellement, des trois réponses signées par $\mathbf{M}$. Bellemare, si intimement mêlé aux transactions relatives à la succession de M. Viger.

[Lettre I]

Mons. Raphaël Bellemare

Université Laval, Québec

5 juin 1903

Montréal

Cher Monsieur,

Vous avez sans doute appris qu'on a trouvé un testament de feu l'abbé Verreau, postérieur à celui qui a été exécuté. Ce testament est de 1873.

Il y a dans ce testament un article qui vous concerne et dont voici la teneur:

«Je donne à mon bon ami, Raphaël Bellemare, Ecuier, l'Album original du Monument de Montréal, le priant de le destiner à sa mort à quelque institution publique. 》

Nous avons cherché cet Album du Monument de Montréal parmi tous les albums de M. Verreau, sans trouver rien qui y corresponde. J'ai demandé au Dr Chabot ${ }^{81}$ si au courant de la Bibliothèque de M. Verreau, et lui-même ne se rappelle pas avoir rien vu de ce genre.

Dans les 28 années qui se sont écoulées entre la date de ce testament et celle de la mort de notre cher ami, M. Verreau a-t-il disposé de cet Album?

Vous devez savoir ce que M. Verreau comprenait par ces mots: "l'Album original du Monument de Montréal ». 
Si vous pouvez nous donner quelques indications qui nous permettent mieux de le reconnaître, nous ferons de nouvelles recherches.

Si non, cher Monsieur, il vous faudra faire votre deuil de ce souvenir que vous destinait votre bon ami.

J'ai l'honneur d'être, cher Monsieur,

Votre très humble serviteur,

Thos.-E. Hamel, p.a.

Procureur de Dlle Justine Verreau,

Exécutrice-testamentaire.

[Lettre II]

Monsieur R. Bellemare

Québec, 25 juin 1903

Montréal

Cher Monsieur,

Je m'empresse de vous remercier de votre bonne et très intéressante lettre que je suis loin d'avoir trouvée trop longue...

Si je l'eusse reçue avant d'avoir connu le 2e testament de M. Verreau, je vous avoue qu'elle m'aurait donné des scrupules à propos des manuscrits du Commandeur Viger. Mais le testament de $1873 \mathrm{me}$ rassure.

En cas que vous n'ayez vu de ce testament, que le paragraphe qui vous concerne et dont je vous ai donné copie, je vais vous copier pour votre satisfaction et pour la nôtre, le paragraphe qui nous concerne.

«Je donne et lègue au Séminaire de Québec les portraits à l'huile de Montcalm, de Wolfe, de trois MM. LaCorne, tous mes manuscrits, tant ceux qui proviennent de $M$. Viger, que ceux que j'ai achetés, excepté ceux de LaFontaine; de plus, toute ma bibliothèque américaine, livres, brochures, cartes, portraits, photographies, les journaux reliés et non reliés, aux conditions suivantes ....

Comme vous voyez, jusqu'à preuve juridique du contraire, les manuscrits de M. Viger nous appartiennent bien. 
Quant aux manuscrits de M. LaFontaine, il [M. Verreau] les léguait au juge Beaudry [sic], à condition que celui-ci, à sa mort, les passerait à la Société historique. Je me suis empressé d'en notifier votre Président, l'honorable juge Baby, lui demandant où, à qui, et par quelle voie, je devais les envoyer. Je n'ai pas encore reçu de réponse, à ce sujet, bien que je lui aie écrit depuis plusieurs semaines.

Très heureux de cette circonstance qui m'a permis d'entrer en relation avec vous, j'ai l'honneur d'être bien respectueusement,

Cher Monsieur,

Votre très humble et tout dévoué serviteur,

Thos.-E. Hamel, p.a.

[Lettre III]

Mons. R. Bellemare, Montréal

\section{Université Laval,} Québec, 18 juillet 1903

Cher Monsieur,

Votre bonne lettre d'hier est arrivée juste au moment où je me disposais à vous écrire.

Le bon M. Dubois, ${ }^{82}$ dans le brouhaha de l'arrivée des trois caisses [des papiers de LaFontaine] et de leur déballement a probablement oublié d'en accuser réception, et je commençais à être en peine. Voilà pourquoi je voulais vous écrire.

Votre lettre a donc été à ce point de vue un grand soulagement.

Maintenant, cher Monsieur, je ne saurais trop vous remercier pour les explications que vous avez pris la peine [de me donner] au sujet des manuscrits Viger.

De mon côté, je ne pouvais douter de l'entière honnêteté de M. Verreau; de l'autre, il me faisait peine de penser à ce point d'interrogation qui devait nécessairement rester dans votre esprit. - Votre lettre a donc été à ce point de vue aussi, un grand soulagement. J'en suis heureux pour vous comme pour moi. 
Agréez, cher Monsieur, l'assurance de ma vive reconnaissance et de la haute estime avec laquelle

J'ai l'honneur d'être bien respectueusement

Thos.-E. Hamel, p.a.

[Lettre IV]

Mons. R. Bellemare,

Québec, 28 juillet 1903

Montréal

Cher Monsieur,

Votre lettre du 23 de ce mois [de juin] est venue après mon départ pour la campagne où j'ai passé quelques jours. J'y réponds à mon retour.

Je suis heureux de vous dire qu'il ne m'est pas venu un seul instant à la pensée que vous vinssiez à douter de l'honnêteté de M. Verreau, et aucune de vos lettres ne pouvait conduire à semblable interprétation. C'est plutôt moi qui me suis mal exprimé et qui ai pu vous donner cette pensée.

J'ai voulu parler du doute que vous pouviez avoir de la réalité du droit de M. Verreau sur les manuscrits Viger, tout en admettant sa parfaite bonne foi dans son erreur, s'il y en avait eu. - Je n'ai pas eu d'autre chose dans l'esprit.

Je ne vous en remercie pas moins des explications que vous avez eu la bonté de me donner.

\section{J'ai l'honneur d'être \\ Cher Monsieur, bien respectueusement \\ Votre très humble serviteur Thos.-E. Hamel, p.a.}

Sans doute ceux qui ont étudié de près cette question du legs de l'abbé Verreau au Séminaire de Québec, savent quelle importance il faut attacher au témoignage de $M$. Bellemare en la matière. Or, ce témoignage, bien élaboré, on le trouve dans les réponses de M. Bellemare aux lettres de Mgr Hamel. Sontelles encore inédites ces pages épistolaires du collaborateur par 
excellence de Jacques Viger, puis de l'abbé Verreau ? Il serait intéressant qu'elles soient publiées lorsqu'une occasion favorable se présentera. ${ }^{83}$ Nous devons bien cela, dirais-je respectueusement, à la mémoire de $\mathrm{M}$. Bellemare, de $\mathrm{Mgr}$ Hamel également. Avec 1903 et 1904, nous en sommes aux dernières années de M. Bellemare. Sa constitution vigoureuse lui fait supporter mieux que tant d'autres le poids des ans. Il reste droit, et sa démarche plus lente ne fait qu'ajouter à la dignité naturelle de sa tenue. L'esprit demeure d'une merveilleuse lucidité. En 1904 la publication de son livre sur les Bases de l'histoire d'Yamachiche le comble de satisfaction. Ne l'a-t-il pas dédié aux habitants de son cher village natal. «A titre de descendants ou de successeurs des pionniers de 1700 , ils ont droit, écrit-il, à cette considération et aussi comme les plus intéressés, à bien connaître les événements du passé concernant leur paroisse ... »

La riche documentation de cet ouvrage témoigne de l'excellente méthode historique de l'auteur qui n'a vraiment rien négligé pour assurer la solidité de la thèse qu'il y soutient. Écoutez ceci : «En commençant mes recherches, écrit M. Bellemare (à la page $29 \mathrm{du}$ Supplément à son ouvrage) sur la paroisse d'Yamachiche, en 1900, le greffe de Cusson fut le premier que je demandai et cherchai dans les voûtes pour y trouver les informations sur les ancêtres de plusieurs familles de notre paroisse. J'ai dû me contenter de prier M. Meilleur Barthe, l'estimable et complaisant conservateur des Archives de ce district [des Trois-Rivières] de ne pas oublier que ce greffe devait avoir été déplacé et oublié quelque part. Je félicite M. Barthe d'avoir eu le bonheur de le retrouver en grande partie l'année dernière [1902 ?] avec l'espérance de découvrir bientôt les séries qui manquent. »

Quelle vision attachante n'avons-nous pas sous les yeux! Un octogénaire, chapeau bas, un manuscrit en main, courbe sa haute taille et pénètre, un peu frémissant à l'idée d'une découverte possible, sous les voûtes d'un bureau de vieilles archives. Notons aussi que les Bases de l'histoire d'Yamachiche furent publiées sous les auspices de la Société historique. La page du titre s'orne des armoiries de ce groupement d'historiens. Voici dans quelles circonstances il en fut décidé: «Je puis assurer M. D.... (un 
des rares détracteurs de cet ouvrage, du moins dans sa thèse fondamentale) que les démonstrations ci-dessus ont pleinement convaincu d'autres savants très compétents en cette matière, et notamment feu M. l'abbé Verreau [le manuscrit était donc achevé dès l'été de 1901], président de la Société historique de Montréal, très versé dans l'interprétation des vieilles écritures et très scrupuleux sur le point de la vérité en histoire. Avant la publication de mon livre... il avait eu la complaisance d'en prendre connaissance et de me prier ensuite de le publier sous les auspices de notre Société historique (voir la page 29 du Supplément). »

Et le bibliophile, nous demandez-vous, que devenait-il ? J'y arrivais justement, ayant l'intention de terminer ce portrait d'un Canadien que trop d'ombre envahissait, par le culte qu'il garda toujours pour les beaux livres. Il portait une attention particulière aux rayons où voisinaient les canadiana ou les americana.

Depuis longtemps, son «trésor des livres rares 》 allait en augmentant. Dès 1892 des écrivains soulignaient les richesses de sa collection de livres dans leurs articles. On le consultait beaucoup. Voici la lettre que lui adressait, en 1894, nul autre que l'abbé J.-A.-Irénée Douville, supérieur et préfet des études du Séminaire de Nicolet.

Monsieur R. Bellemare,

[Séminaire de Nicolet,] 27 mars 1894

Cher Monsieur,

A mon dernier voyage à Montréal, $j$ 'avais fait le projet, avec mon ami le Dr Desjardins, d'aller passer une soirée chez vous. Une affaire est survenue pour nous empêcher d'avoir ce plaisir. J'espère que je me reprendrai au mois de mai. En attendant, je me permets de vous consulter sur les meilleurs moyens à prendre pour compléter notre bibliothèque canadienne. Plus j'y travaille, plus j'ai du plaisir à le faire. Je sais que vous êtes un maître en tout cela et vous ne me refuserez pas vos conseils. Il me semble que la réputation de notre maison recevrait un joli accroissement si l'on pouvait dire qu'elle 
possède une des meilleures et des plus complètes bibliothèques canadiennes du pays.

Ceci posé, veuillez donc me dire, s'il vous plaît, où je puis tenter la fortune pour avoir les ouvrages suivants :

- Le Routier du pilote Jean Alphonse

- Jean de Laêt « Novus orbi » ou la traduction

- Hakluyt. The principal navigation, etc.

- Purchas. « Hakluytus Porthumus », 1617-1625

- Ramusio

- Le P. Ch. Leclercq. Etablissement de la Foi

- Du Creux. Hist. Canad.

- Denys. Descr. géogr. et hist. des Côtes de l'Am.

- Martin (Dom Claude). Marie de l'Incarn.

- Carayon. Premières Missions des Jésuites

- Hutchnivan. History of the Colony of Mass.

Parmi les publications du pays, il en est une que je tiens beaucoup à compléter, c'est celle de la Société historique de Montréal. Nous avons les 1e, 4 e, 5e, 6e, 7e et 8e livraisons des Mém. et Doc. publiés par cette Société. Où puis-je me procurer ceux qui nous manquent?

En voilà bien assez pour aujourd'hui. Pardonnez-moi ma grande liberté, en vous souvenant que je travaille pour votre vieux Collège de Nicolet, sans épargner ses plus anciens et ses plus dévoués amis.

Nous faisons copier toutes les archives de l'Archevêché de Québec qui concernent notre maison. Il y a dix gros volumes.

Recevez l'expression de la sincère et distinguée considération avec laquelle je me souscris,

Votre tout dévoué,

J.-A. Ir. Douville, ptre

Nous savons que Mgr Douville publiait, en 1903, son Histoire du Collège-Séminaire de Nicolet, à l'occasion de son Centenaire. Cette énorme compilation de faits, de portraits, de souvenirs, était présentée dans un style clair et fort agréable. Ce fut avec une joie profonde et les yeux souvent embués que M. Bellemare parcourut ces pages où revivait sa jeunesse étudiante; où il évoquait les figures vénérées de ses vieux maîtres; où tant de 
chers amis disparus revivaient devant lui, joyeux, remplis de beaux projets, si compréhensifs, de cœur et d'esprit. Il avait survécu à tous, et l'absence de certains d'entre eux, les soirs de mélancolie, pesait lourdement sur son cœur. Mais bientôt, il les retrouverait tous, car la fin du voyage approchait pour lui. Comme pour eux, jadis, quelques paroles de saint Jean résonnaient en son âme et conscience: "Et opera sequuntur illos ». Serait-ce pour sa justification? Il avait désiré, certes, bien servir Dieu, le prochain malheureux, sa grande et sa petite patrie. Et comme à l'accoutumée, chez ce vieillard à la foi vivante, réminiscences et réflexions s'achevaient dans un appel humble et confiant au Maître de la vigne.

Le premier février 1906, trois semaines avant de compléter ses 85 ans, "ce fardeau bien lourd à porter», avait-il prononcé, M. Bellemare s'éteignait doucement.

\section{Marie-Claire Daveluy}

\section{NOTES ET RÉFÉRENCES}

1 Madame Léonine-Euchariste Normandin était la seconde femme de M. Bellemare. Il l'épousait le 11 mai 1885. Le premier mariage de M. Bellemare eut lieu le 18 septembre 1849 .

2 Dans l'ouvrage: The Canadian Biographical Dictionary and Portrait Gallery of Eminent and Self-Made Men, Quebec and the Maritime Provinces (Chicago, New York and Toronto, American Biographical Publishing Company, H. C. Cooper \& Co., proprietors, 1882), nous trouvons à la page 55 , une intéressante notice sur M. Bellemare. Quelques détails que je n'ai lus nulle part ailleurs y ont été insérés et m'ont été très utiles. Naturellement, comme en d'autres esquisses biographiques, on loue le bibliophile et le collectionneur de canadiana: «He possesses, a-t-on noté, one of the best private libraries of Montreal, containing a most rare collection of books and documents on the early history of the country. $\gg$ Je me souviens que M. Bellemare avait pu se procurer le rarissime Voyage de Champlain en 1603, paru à Paris en 1604. Philéas Gagnon ne comptait point cet ouvrage dans sa riche collection. La bibliothèque de $M$. Bellemare fut transportée, à sa mort, en 1906, à Ottawa, chez sa fille aînée, Mathilde, Madame D.-L. Desaulniers. Elle comptait plus de 5,000 volumes. Elle fut vendue, peu d'années avant la mort de celle-ci, vers 1941, à un libraire spécialisé en canadiana de Montréal. Quel dommage qu'il ne se trouvât point à l'époque quelque Mécène ou une institution pouvant l'acheter et empêcher ainsi la dispersion de cette admirable collection Bellemare, fruit des recherches de toute une vie. Je le redis, je parle ici en témoin oculaire, car M. Dionis LeSieur-Desaulniers était le frère de ma mère.

3 Deux des albums historiques de M. Bellemare méritent une mention particulière. Le premier est un recueil de documents, d'autographes, d'aqua- 
relles magnifiques et de diverses autres illustrations, constituant tous des pièces originales ou tirées de documents de première main et qui avaient été réunies et annotées par le Commandeur Jacques Viger, premier maire de Montréal (en 1833) et fondateur de la Société historique de Montréal (1857). Le deuxième album contenait 17 aquarelles originales signées par James Duncan de Montréal et concernait nos communautés religieuses de femmes au Canada. Un beau manuscrit autographe de Jacques Viger, donnant des notices historiques et des statistiques sur 17 communautés religieuses de femmes, accompagnait l'album. Madame Desaulniers, à sa mort en 1941, léguait ces albums à ses enfants. J'eus l'honneur de convaincre mes cousines, Mesdames Robert Manion et Eugène Poitevin, d'Ottawa, de vendre à la Ville de Montréal, pour sa bibliothèque, ces trésors artistiques et historiques conservés par leur aïeul. M. Léo-Paul Desrosiers, conservateur de la Bibliothèque Municipale, en 1944, un littérateur et un historien, s'y intéressa et réussit à loger bientôt, dans la Salle Gagnon, qu'il enrichissait sans cesse, le premier de ces Albums. Le deuxième fut acquis, en 1953, par M. Jules Bazin, successeur de M. Desrosiers, un artiste et un bibliophile, qui en reconnut vite la valeur. - Sur le premier album Viger, on lira deux études excellentes: l'une par Maximilien Bibaud, dans son Panthéon canadien (1891), 308-310; l'autre, par l'historien et l'artiste, Mgr Olivier Maurault, p.ss.p.a., parue dans les Cahiers des Dix (année 1944), 83-99. Sur le second album, on consultera le petit ouvrage de Laroche-Héron, pseudonyme du Comte Henri de Courcy, Les Servantes de Dieu en Canada (Montréal, 1855, 158 pages, in-8).

4 Sir Frederick Haldimand (1718-1791) est le quatrième gouverneur général du Canada sous le régime britannique (de septembre 1777 à l'automne de 1784). Je me suis souvent demandé pour quelle raison M. Bellemare avait acquis cette petite toile. C'est qu'un document de première importance, signée par le général Haldimand, en 1781, concerne les co-seigneurs d'Yamachiche, Charles et Julien LeSieur, et par eux, indirectement, les trois colons qu'ils amenèrent en cet endroit, en 1703 . Ces colons, les trois frères Gélinas, s'établirent les premiers sur le site actuel de la ville d'Yamachiche. Le cadet portait le nom de Jean-Baptiste, et prit aussitôt le surnom de Bellemare, l'imposant ainsi, suivant la coutume du temps, à toute sa descendance. Ce Jean-Baptiste Gélinas, dit Bellemare, fut l'ancêtre à Yamachiche de Raphaël Bellemare qui apparaît, pour sa part, à la septième génération.

5 Les lettres autographes dont je parle sont au nombre de 70. La plupart sont signées et datées; les autres faciles à identifier. Leur importance varie, sinon leur intérêt. Elles vont des années 1845 à 1903. La correspondance de M. Bellemare est considérable. Elle a été déposée par un de ses arrière-petits-fils, M. Jacques Manion, à l'époque attaché commercial à l'Ambassade du Canada à Paris, aux Archives Fédérales, rue Sussex, à Ottawa. Elle réserve des surprises aux historiens qui la dépouilleront.

6 Charles Chiniquy (1809-1899) fut un ancien élève et un protégé du Collège de Nicolet. Il fut ordonné prêtre en 1833. Ses relations épistolaires avec $M$. Bellemare ne furent pas de longue durée. La dernière lettre écrite au rédacteur de la Minerve est datée du 4 janvier 1855. Elle vient de Sainte-Anne, Kankakee (Illinois). Chiniquy remercie les propriétaires, et surtout le rédacteur, M. Bellemare, de l'envoi de la Minerve.

7 La Minerve, l'organe du parti libéral-réformiste, soutint Louis-Hippolyte LaFontaine dans ses luttes politiques. Elle fut d'inspiration profondément catholique, avec des directeurs comme Gérin-Lajoie, (1844-1847) et Raphaël Bellemare (1847-1852). La Minerve vécut des heures orageuses à l'époque où M. Bellemare la rédigeait. 
8 Patrie intime fut d'abord le titre du beau poème souvent cité de Nérée Beauchemin. Il a été publié pour la première fois, en 1898, dans le premier volume des Vieilles familles d'Yamachiche, par François LeSieur-Desaulniers. Plusieurs variantes de ce poème parurent dans la suite. Enfin, en 1928, Nérée Beauchemin réunit sous le titre de Patrie intime, les écrits poétiques de sa maturité.

9 Voir les Vieilles Familles d'Yamachiche par François Le SieurDesaulniers, tome second (Montréal, Beauchemin, 1899). Discours préliminaire par Raphaël Bellemare, p. XXX.

10 Ibid: XIX.

11 Ibid: XXVII.

12 Voir Histoire de la paroisse d'Yamachiche (précis historique), par l'abbé N. Caron, ptre, chanoine, curé de Maskinongé. Supplément par Frs. L. Desaulniers, avocat, député au Parlement fédéral. Chapitre spécial par Benjamin Sulte. (Trois-Rivières, P.-V. Ayotte, libraire-éditeur, rue NotreDame, 1892), 129-132. note 1 .

13 F.-L. Desaulniers, Les Vieilles Familles d'Yamachiche, II: 70-75,

14 « Pierre Laviolette, nous apprend Monseigneur Douville, dans son Histoire du Collège-Séminaire d’e Nicolet (2 vol., Montréal, 1903), I: 102103; II: 11-12, était né le 5 mars 1774, à Boucherville. Il fit ses études au Collège de Montréal et y prit la soutane. En 1816-1817 et en 1817-1818 il professa les Belles-Lettres et la rhétorique au Collège de Nicolet. Il quitta le Séminaire en 1818. Il épousa la fille du seigneur des Mille-Iles, à SaintEustache, Elmire Lambert-Dumont. Il devint plus tard, en 1825, le propriétaire de cette seigneurie. [Il fut l'] auteur de: «O Nicolet, qu'embellit la nature ... \& écrit en 1821, lors d'une visite au Collège qu'il n'avait jamais oublié. On y conserve le manuscrit original de cette pièce de vers. Pierre Laviolette «fonda, de sa bourse privée, à Saint-Eustache, un collège où bon nombre de jeunes gens firent leurs études et devinrent plus tard des citoyens marquants. [II] était un ami passionné des lettres, un poète distingué... Il reste de lui de nombreuses poésies, des drames et d'autres écrits sur divers sujets. M. Laviolette mourut à Saint-Eustache en $1854 \ldots$ Il était âgé de 60 ans. 》 (Voir Le Répertoire national... compilé par Joseph Huston, Montréal, 1893, 2e éd. illustrée de 50 portraits... I: 261-262, note 1).

15 Le poète Louis Fréchette, élève à Nicolet vers 1860 , écrivit son poème « les Pins de Nicolet » en 1861. L'historien du Collège, l'abbé Douville, le reproduit en entier dans son ouvrage (appendice du Chapitre II, I: 65).

16 L'abbé Joseph-Sévère-Nicolas Dumoulin est né à Sainte-Anne duBout-de-l'Ile de Montréal, (aujourd'hui Sainte-Anne-de-Bellevue), le 5 décembre 1793. «Il passa son enfance dans une jolie maison en pierre qui se trouve à quelques arpents au bas de l'église ...; i et ses années d'adolescence, à St-Zéphirin de Courval où son père était seigneur. » Il fit ses études au Collège de Nicolet, et fut ordonné en 1817. Vicaire pendant une année à Québec, il partit avec Mgr Provencher pour aller ouvrir les missions de la Rivière-Rouge. En 1824, revenu de la Rivière-Rouge, il exerça le ministère à Saint-François de la Rivière-du-Sud. En 1825, il était curé d'Yamachiche et le demeura jusqu'à sa mort. Il mourut aux Trois-Rivières, où il était en repos chez son frère, le 27 juillet 1853, à l'âge de soixante ans. Nul curé d'Yamachiche, peut-être, ne fut vénéré et aimé à l'égal de M. Dumoulin.

17 Louis-Marie Brassard, curé de Nicolet (de 1750 à 1800) était né à Québec le 18 décembre 1726. Il fit au Séminaire de Québec son cours classique, puis sa théologie et fut ordonné prêtre le 21 décembre 1749. "Après la construction d'une [ nouvelle] église, M. Brassard songea à doter sa 
paroisse d'une école élémentaire gratuite... \ Il ne put réaliser ce projet de son vivant. Mais en laissant par testament une maison de pierre, quelques rentes et un terrain qui seraient affectés à cette fondation, il permit à Monseigneur Denaut, évêque de Québec, qui devenait finalement propriétaire du legs du curé Brassard, de fonder et d'ouvrir une école latine en l'année 1804. On considère cette époque comme celle du commencement du Collège de Nicolet (Mgr Douville, Histoire du Collège-Séminaire de Nicolet, (2 vol., 1903), I: 1-18).

18 Raimbault (abbé Jean) est né à Orléans en France, le 4 février 1770. Il fut un des jeunes ecclésiastiques que la Révolution française conduisit en notre pays. Arrivé à Québec, le 6 juillet 1795, alors qu'il n'était que simple tonsuré, il professa d'abord la philosophie au Séminaire de Québec. Puis on le vit successivement, une fois ordonné prêtre, curé de l'Ange-Gardien, en 1797; curé de la Pointe-aux-Trembles de Montréal, en 1805; et finalement, curé de Nicolet et supérieur du Séminaire, derniers postes qu'il occupa jusqu'à sa mort, le 16 février 1841. Il était âgé de 71 ans. Le Collège-Séminaire de Nicolet possède le crâne de ce bienfaiteur insigne de l'Institution.

19 Joseph-Onésime Leprohon, né à Montréal, le 16 février 1789, était le fils de Jean-Philippe Leprohon et de Marguerite Parent. Ordonné le 6 février 1814, il fut d'abord vicaire à Deschambault (1814), à Belœil (18141816), avec desserte de Saint-Hilaire-sur-Richelieu. Puis il devint directeur du Collège-Séminaire de Nicolet de 1816 à 1841 . Il mourut curé de Nicolet (1841-1844), le 19 mars 1844. Il comptait 55 ans d'âge. Son crâne est aussi conservé au Séminaire de Nicolet. «Tous ceux qui ont été sous lui, élèves et professeurs, en ont fait les plus grands éloges comme directeur. 》 Voir ibid., 6 .

20 Paul-Loup Archambault est né à la Rivière-des-Prairies, près de Montréal, le 29 septembre 1787. Il fit ses études à Montréal et à Nicolet et fut ordonné à Québec par Mgr Plessis, le 18 octobre 1812. Il fut vicaire aux Cèdres de 1812 à 1813 , professeur de 1809 à 1811 , puis directeur du Séminaire de Nicolet de 1813 à 1816. Nommé curé de Vaudreuil en 1816, il le demeura durant 42 ans. Il y mourut le 26 février 1858, âgé de 70 ans. Il encouragea beaucoup Mère Marie-Anne dans sa fondation des Sœurs de Sainte-Anne. $\mathrm{I}: 100$.

${ }^{21} \mathrm{Mgr}$ Douville, Histoire du Collège-Séminaire de Nicolet (1903),

22 Thomas Caron est né à Louiseville, comté de Maskinongé, le 19 juin 1819. Il fit ses études à Nicolet et fut ordonné le 27 août 1842. Il professa de longues années à Nicolet, y fut aussi directeur des élèves, préfet des études, directeur des ecclésiastiques. Il occupait encore la chaire de théologie (1872-1878) l'année de sa mort, arrivé le 24 septembre 1878, à l'âge de 59 ans. L'abbé J.-B.-A. Allaire, dans son Dictionnaire biographique du Clergé canadien (Montréal, 1910), II: 98-99, déclare: "Il a laissé une mémoire impérissable chez tous ceux qui ont vécu sous lui ou avec lui. »

${ }^{23} \mathrm{Mgr}$ Joseph Signay est né à Québec le 8 novembre 1778. Il fut ordonné à Longueuil par Mgr Denaut. Après avoir exercé son ministère, soit comme vicaire, soit comme curé, en plusieurs paroisses des régions de Québec et de Montréal, il devint curé de la Cathédrale de Québec, puis coadjuteur de l'Archevêque de Québec. Sacré évêque par Mgr Panet, le 20 mai 1827 , il succéda à celui-ci en 1833 . Il fut un ami et un protecteur du Collège de Nicolet. Il mourut le 3 octobre 1850, âgé de 72 ans.

24 La tragédie en vers d'Antoine Gérin-Lajoie fut donc jouée pour la première fois, au Collège de Nicolet, en juin 1844. Combien de fois ne sera-t- 
elle pas reprise par la suite. Lareau, dans son Histoire de la littérature canadienne (Montréal, 1874), 72-74, en parle avec bienveillance, en somme, malgré quelques critiques. «Il y a des beautés de détails, beaucoup même, écrit-il, mais l'auteur n'a pas donné assez d'extension à son thème, etc. 》 On trouve le texte en entier de la tragédie dans le Répertoire national de Huston, II : 3-55.

25 Jacques-A. Tailhade était un Français établi au Canada où il s'était fait naturaliser. Il étudia le droit à Montréal dans les bureaux de John Boston et Charles-Clément de Sabrevois de Bleury. Admis à la pratique du droit le 28 septembre 1841, il devint en peu de temps un avocat réputé. Il offrait bientôt de fonder une école de droit à Montréal, mais le projet n'eut pas de suite. Il mourut en 1852 .

N.B. Je tiens ces renseignements d'un bibliothécaire de la Bibliothèque du Barreau à Montréal, M. Arthur Perrault, que je remercie pour sa courtoisie et son obligeance. On consultera sur M. Tailhade, pour quelques détails supplémentaires, les remarquables études sur les Avocats de Montréal ainsi que sur l'histoire du Barreau de Montréal, publiées dans les Cahiers des Dix, par le Juge Maréchal Nantel. Aussi le Bull. des recherches historiques, XXXXVII : 237.

${ }^{26}$ L'on comprend que Raphaël Bellemare s'intéressait au mouvement politique du jour. « Le moment était critique, déclare Léon Gérin, dans la biographie de son père, Antoine Gérin-Lajoie. Les Chambres allaient être dissoutes, on se préparait à des élections. » Le nouveau gouverneur, lord Elgin, venait d'arriver... \& La Fontaine et Baldwin, qu'on avait tenus à l'écart du pouvoir depuis 1843 , allaient triompher cette fois et se voir rappelés au Parlement pour y former un nouveau Ministère. 》 (Voir Léon Gérin, Antoine Gérin-Lajoie ... (Edition du Centenaire, Montréal, Le Devoir, 1925), 74).

27 Dans le même ouvrage, Léon Gérin note quelques détails sur l'entrée de M. Bellemare à la Minerve. "Il [son père] négocia l'affaire, tant avec Bellemare qu'avec Duvernay. Le 18 août [1847] il présentait Bellemare au propriétaire de la Minerve... Dès la fin de la première semaine [Raphaël Bellemare] touchait cinq piastres [sic], chiffre que Gérin-Lajoie [entré à deux dollars par semaine] avait pris un an à atteindre. Antoine GérinLajoie, ajoute dans ses Mémoires, que son fils cite abondamment: "Duvernay fut bientôt satisfait de Bellemare et n'aurait pas voulu l'échanger pour moi.» L'on était loin de la sinécure à cette époque où la tâche de rédacteur était écrasante. Il fallait être à la fois prote et compositeur, correcteur d'épreuves, traducteur, etc. Cet état de choses que nous rapporte Léon Gérin, je le voyais récemment confirmé par les souvenirs sur son grandpère Bellemare, que nous racontait son petit-fils, M. Paul Desaulniers d'Ottawa, fils de D. L.-Desaulniers et de Mathilde Bellemare. Il demeura quelques années sous le toit de son aĩeul avec lequel il causait volontiers de ces temps difficiles.

28 « Mon principal but en quittant la Minerve, narre Antoine GérinLajoie dans ses Mémoires (ibid., 71), avait été de me livrer entièrement à l'étude de ma profession [d'avocat] ... Pendant les mois de septembre et d'octobre, continue-t-il (ibid., 82), je dépouillai plus de deux cents dossiers... j'avais de longues conversations sur le droit avec mon ami Lafrenaye....

29 Aegidius Fauteux, dans sa vivante étude sur Le Duel au Canada (Montréal, les Editions du Zodiaque, [1934], 172-173), raconte au long le fameux duel de Ludger Duvernay avec Charles Clément de Sabrevois de Bleury «qui avait été violemment pris à partie par la Minerve. Outragé, 
le député [Sabrevois de Bleury] exigea une rétractation....» ou le duel. Il eut lieu le 17 avril 1836. «Duvernay [ayant été] blessé, les parties quittèrent le terrain sans plus d'explications. 》 Tout de même, le propriétaire de la Minerve ne manquait point de cran d'accepter une rencontre avec un adversaire bon tireur au pistolet, lui qui l'était si peu.

$30 \mathrm{M}$. Bellemare entrait certainement à la Société Saint-Jean-Baptiste peu de semaines après son installation à la Minerve. Il en avait été ainsi, du reste, avec son prédécesseur. Gérin-Lajoie travailla durant quelques années au secrétariat de la Société. Habite-t-on impunément sous le même toit qu'un fondateur! Duvernay avait du flair. Il savait dépister des membres intelligents et patriotes.

31 Charles-Joseph Coursol, une figure haute en couleur aux temps orageux de l'Union, naquit à Amherstburg, Haut-Canada, le 3 octobre 1819 . Par sa mère, née Mélanie Quesnel, il était le petit-fils de Joseph Quesnel, poète, dramaturge et musicien. Son père mourut fort jeune et CharlesJoseph fut adopté par son oncle, Frédéric-Auguste Quesnel, qui avait amassé une fortune dans le commerce des fourrures. Admis au Barreau, en 1841, Charles-Joseph Coursol se préoccupe beaucoup plus de la politique que de l'exercice de sa profession. La carrière de ce disciple impétueux et fidèle de LaFontaine a été fort bien narrée par M. Léon Trépanier, dans les Cahiers des Dix (Montréal, 1957, no 22). Sur toute cette époque de luttes en divers domaines, on consultera surtout le Chanoine Groulx dans sa série d'ouvrages intitulés: Notre Mầtre, le Passé. Notre grand historien y a tracé d'émouvants portraits de LaFontaine et d'Augustin-Norbert Morin, entre autres. Charles-Joseph Coursol, dynamique et bretteur, y revit bien en forme et de façon sympathique. A lire aussi le Duel au Canada d'Aegidius Fauteux.

32 Comme cet article fait partie du Supplément à l'Histoire d'Yamachiche, de François LeSieur-Desaulniers, il est probable que celui-ci fit luimême la traduction du texte anglais de G. Rose, en 1888. Remarquons aussi que ce dernier auteur a utilisé largement les notes biographiques parues six ans plus tôt dans The Canadian Biographical Dictionary, ouvrage que nous citons dans la note 2.

33 Le journal L'Avenir fut fondé à Montréal le 16 juillet 1847, par Jean-Baptiste Eric Dorion et George Batchelor. Il dure jusqu'en 1852, reprend en 1854, mais pour ne durer que peu de temps. (Voir NarcisseEutrope Dionne, Inventaire chronologique des livres, brochures, journaux et revues... (Québec, 1905), I: 145.

${ }^{34}$ Le Moniteur canadien, fondé à Montréal le 12 octobre 1849, par J.-G. de Montigny (ibid., 146).

35 Le Pays, fondé à Montréal le 15 janvier 1852, par J.-A. Plinguet, et rédigé par Louis-Antoine Dessaulles et Labrèche-Viger, était reconnu comme le journal des intérêts démocratiques. Il y eut deux éditions, hebdomadaire et tri-hebdomadaire. Un anticléricalisme regrettable s'y fit bientôt jour. Il disparaît en 1871 (Ibid).

36 Joseph Doutre, né à Beauharnois en 1825, fit ses études au Collège de Montréal. Il fut admis au Barreau en 1847. On le regarde comme «l'un des plus célèbres dirigeants du parti rouge ». Son duel avec Sir GeorgesEtienne Cartier a été raconté avec beaucoup de verve par Aegidius Fauteux, dans son Duel au Canada (op.cit., 272-278). Joseph Doutre se révolte ouvertement contre les ordonnances de Mgr Bourget « au sujet des ultralibéraux. L'affaire Guibord le porta aux extrêmes exaspérations. 》 (Louis Le Jeune, Dictionnaire général du Canada (Ottawa, 1931), I: 530-531). Il fut l'un des principaux rédacteurs du journal l'Avenir, avec Charles Daoust, 
son beau-frère, et Jean-Baptiste Eric Dorion, le fondateur de cette feuille, que tous appelaient «l'Enfant terrible», à cause de sa violence et de son physique malingre. Joseph Doutre fut aussi un des collaborateurs du Pays. On lui doit un roman, Les fiancés de 1812 (1844), et une nouvelle en 1846, parue dans le Répertoire national de Huston, III: 335. Il mourut à Montréal, le 3 février 1886, âgé de 61 ans.

37 Pierre Blanchet que M. Fauteux appelle un des piliers de l'Avenir et le plus fougueux des républicains de son temps, se vantait d'être le petitfils des terroristes de 1793. On l'appelait «le citoyen Blanchet». Il est né, d'après les recherches exactes de Pierre-Georges Roy, à Saint-Pierre de la Rivière-du-Sud, le 8 octobre 1812. Ses études classiques terminées il porta la soutane durant un an. En 1846, il fut admis au Barreau, mais préféra à cette profession celle du journalisme. Il collabora à l'Avenir, mais peu à peu ses idées exaltées lui nuirent. Ses amis l'abandonnèrent. Il devint cultivateur dans un rang situé près d'Arthabaska. Malade, vieilli, des voisins le conduisirent à l'Hôtel-Dieu d'Arthabaska. Il s'y convertit. (PierreGeorges Roy, Toutes petites choses du régime anglais, 2e série, (Québec, Ed. Garneau, 1946), 212-213). Henri d'Arles a raconté sa fin édifiante qu'il termine par ces mots: « $O$ mystère inouï, éternellement recommencé de la grâce. Le "Citoyen Blanchet 》 est mort comme un saint. 》 (Bulletin des recherches historiques (1925), 17).

38 Jean-Baptiste Eric Dorion, un des frères de Sir Antoine-Aimé Dorion, n'avait ni le grand talent ni la distinction de celui-ci. Mais il ne manquait point de courage. Malgré sa petite taille et sa mine misérable, il s'offrit un jour pour croiser le fer avec le vigoureux athlète, Sir GeorgesEtienne Cartier, en remplacement d'un collaborateur absent de l'Avenir (Joseph Doutre). Qui s'étonnera que Cartier, en colère, et avec raison ce jour-là, l'ait dédaigné comme adversaire sur le terrain.

39 Louis-Antoine Dessaulles (1819-1895) fut d'abord médecin, puis Conseiller législatif. Démissionnaire de ce dernier poste en 1868 , il adhéra au parti rouge et devint l'éditeur du Pays. Le Chanoine Groulx, dans la 3e série de Notre Maître le Passé, a rappelé la carrière et les écarts si pénibles de ce journaliste envers les autorités religieuses et même envers le Pape. Avec sa vision lucide des hommes et des faits, le Chanoine a dépeint parfaitement l'atmosphère de ces temps de violences verbales et de tragiques événements politiques. Greffier de la Couronne et de la Paix jusqu'en 1875, M. Dessaulles mourut à Paris, le 5 août 1895 . Il comptait 76 ans.

40 La haute et dominante figure de Sir Georges-Etienne Cartier (18141873) est trop bien connue pour que j'y insiste ici. Cet ami de la Minerve, de ses rédacteurs, Gérin-Lajoie et Bellemare, et de son propriétaire, Ludger Duvernay, donna maintes preuves de sa sincérité à leur endroit. Sans doute, évoquait-il parfois, auprès de Duvernay, les temps heureux de sa jeunesse, alors qu'il adhérait aux sentiments patriotiques du fondateur de la Société Saint-Jean-Baptiste, l'accompagnant au banquet de fondation et y chantant une pièce de sa composition: «Mon pays, mes amours». Cartier fut un fidèle serviteur, héroïque à certaines heures, de ce Canada dont il a finalement bien dirigé les destinées aux jours de la Confédération. Il mourut à Londres, le 20 mai 1873 et fut inhumé à Montréal, le 30 juin suivant.

41 Louis-Victor Sicotte (1812-1889) avocat, puis ministre sous l'Union avant de devenir, en 1863, juge de la Cour Supérieure pour le district de Saint-Hyacinthe, fut lui aussi, un ami fidèle de la Minerve et de ses rédacteurs, Gérin-Lajoie et Bellemare. Il eut la joie, en 1859, avant de quitter le Ministère des travaux publics, qu'il dirigea de 1857 à 1859 , de réussir à faire nommer Antoine Gérin-Lajoie à la Bibliothèque du Parlement où il 
se chargerait tout particulièrement de la section française. Dans ses Mémoires, Gérin-Lajoie parle souvent de ce politique distingué et ne le nomme jamais alors que «mon ami Sicotte ». Le juge Sicotte décéda à SaintHyacinthe le ler août 1889 .

42 L'archiviste montréalais, Edouard-Zotique Massicotte (décédé en 1947), a publié dans le Bulletin des recherches historiques (vol. XXVI, no 1, janv. 1920) l'acte de «Vente du titre de la Minerve, par Augustin-Norbert Morin à Ludger Duvernay, le 18 janvier 1827, 》 en l'accompagnant de quelques notes et détails. La Minerve fut achetée par Duvernay, pour et moyennant la somme de sept livres, dix chelins courant... La feuille comptait alors 300 abonnés. M. Morin s'engageait à l'éditer et à la rédiger pour six mois, moyennant le maigre salaire de 15 livres courant (environ 70 dollars). Il était alors étudiant en droit, comptait 24 ans, et avait fondé la Minerve un an plus tôt. La Minerve cessa de paraître en 1899.

43 Voici ceux qui précédèrent $M$. Bellemare à la rédaction de la Minerve, d'après une liste écrite de sa main, que j'ai en ce moment devant les yeux: A.-N. Morin, 1827-1830; Léon Gosselin, 1830-1835; Leblanc de Marconnay, dates omises; N. Aubin, dates omises; Pottel et Amyot, dates omises; John Phelan, dates omises; Antoine Gérin-Lajoie, 1844-1847; Raphaël Bellemare, 1847-1855. Si M. Bellemare a inscrit l'année 1855 au lieu de l'année 1852 comme étant celle où il abandonna la rédaction de la Minerve de façon officielle, c'est que durant ses études de droit, il fut un rédacteur intermittent, mais se rendit tout de même au journal assez fréquemment. Le dernier rédacteur que mentionne $\mathbf{M}$. Bellemare est Jules-Paul Tardivel. A la suite de la liste des rédacteurs, sous la rubrique « Contribution volontaire à certaines époques », on lit les noms de Sir Louis-Hippolyte LaFontaine, MM. Girouard, Rodier, Hector-L. Langevin, Pierre J.-O. Chauveau, Thomas-J.-J. Loranger, Joseph-Charles Taché, A. Courcy de la Roche-Héron, J.-U. Beaudry.

44 L'histoire de ces temps difficiles pour les étudiants en droit a été narrée récemment par deux érudits de la Société des Dix, MM. FrancisJoseph Audet et le juge Maréchal Nantel.

45 Francis-Joseph Audet (1867-1943), chercheur infatigable et heureux, a vu ses nombreux écrits, compilés, annotés et scientifiquement décrits dans la bio-bibliographie exhaustive que lui a consacrée son disciple et collaborateur, au bureau des Archives fédérales, à Ottawa, M. Lucien Brault. Dans les Cahiers des Dix, car M. Audet fut membre de la Société des Dix dès la fondation, en 1935, il a présenté sept études historiques, parmi lesquelles nous avons consulté avec profit, les « Débuts du Barreau de la Province de Québec (1937) 》 et un portrait de Charles-Clément de Sabrevois de Bleury (1940).

46 Le juge Maréchal Nantel (1891-1956) a laissé de nombreux travaux sur l'histoire du Barreau au Canada et sur diverses autres questions de droit. Ils ont paru dans la Revue $d u$ droit dont il fut l'un des fondateurs, et dans les Cahiers des Dix. Il était membre de la Société des Dix depuis 1940 et publia, de 1940 à 1952 , douze études d'une rare érudition légale. Le juge Nantel écrivait avec goût, mesure et correction. Les connaissances juridiques de cet ex-bibliothécaire de la Bibliothèque du Barreau étaient le fruit de vastes lectures et de recherches poursuivies sans relâche dans un domaine spécialisé et peu exploré.

47 Je crois que si les cousins Antoine Gérin-Lajoie et Raphaël Bellemare ne se virent pas dans la nécessité d'étudier un an chez des avocats de langue anglaise, c'est que tous deux étaient déjà d'excellents bilingues. Ils pouvaient certes plaider en l'une ou l'autre langue. Voilà un des avantages 
que leur procuraient alors, les méthodes pédagogiques à la fois scientifiques et pratiques, qu'ils s'agisse de linguistique, d'histoire ou de polémique religieuse, de leur incomparable maître et ami, l'abbé J.-B. Antoine Ferland.

48 Voir les Cahiers des Dix (1942), VII: 185-213.

49 Pierre-Richard Lafrenaye est né aux Trois-Rivières, le 12 juin 1824. Elève au Collège de Nicolet dès sa dixième année, en 1834, il en sortait après sept ans d'études. Il fut admis au Barreau en 1845. Il fut bientôt reconnu comme un excellent légiste. Il devint secrétaire du Barreau (1851-1852); membre du Comité de la Bibliothèque (1854-1860 et 1861-1869); conseiller au Conseil général du Barreau (1855-1859, 1862-1865 et 1869), trésorier du Barreau (1859); examinateur au Conseil du Barreau (1855); conseiller de la Reine (1855). En somme, une des brillantes personnalités judiciaires de l'époque. Un excellent orateur, en outre, que l'Institut Canadien, au temps de la présidence de Gérin-Lajoie, invitait et applaudissait. Il décédait malheureusement à l'âge de cinquante ans, alors que sa renommée le plaçait au premier rang des jurisconsultes de son temps.

N.B. Nous devons de nouveau ces précieux renseignements à M. Perrault, bibliothécaire à la Bibliothèque du Barreau de Montréal. Nous lui en exprimons notre reconnaissance. (Voir aussi Edmond Lareau, Histoire de la littérature canadienne (1874), 389, qui rappelle que Pierre-Richard Lafrenaye fut un des fondateurs, en 1857 , de la revue Lower Canada Jurist. Dans cette collection, les décisions des tribunaux, les textes sont écrits indistinctement dans les deux langues, selon que la cause est rapportée par un avocat français ou anglais.

50 Voir les Cahiers des Dix, (1942), VII: 185-213, l'article du juge Nantel sur les « Avocats à Montréal 》.

51 Alfred Duclos DeCelles (1843-1925), bibliothécaire du Parlement de 1855-1925, écrivait son article sur Gérin-Lajoie, à la demande de François L.-Desaulniers, qui désirait l'insérer dans le Supplément qu'il préparait pour l'Histoire d'Yamachiche du chanoine Caron (Voir cet ouvrage déjà cité, 257-260).

52 Qui ne comprend la sympathie manifestée à Madame Bellemare par Antoine Gérin-Lajoie, au lendemain du terrifiant incendie à Montréal, en 1852 ? Le Père Langevin, jésuite, en a fort bien donné l'impressionnant récit dans sa Vie de Monseigneur Bourget (Montréal, 1931), 181-183. « En une demi-journée, a-t-il écrit, la Cathédrale Saint-Jacques (rue SaintDenis, alors) et les deux palais de l'évêché étaient anéantis ... Après cinq heures d'incendie le quadrilatère Saint-Laurent-Vitré, Saint-Denis-CoteauBarron était aux trois quarts consumé... Le soir, le feu se ralluma près du Champ-de-Mars, et cette fois tout le faubourg Québec fut rasé... L'incendie ne fut maitrisé que vers huit heures le lendemain matin. La catastrophe laissait sur le pavé mille sept cent vingt-sept familles, c'est-à-dire neuf mille personnes. $\gg$

53 Le filleul dont parle tendrement le cousin Antoine ne vécut pas treize mois. Philippe-Oreste-Marie Bellemare, baptisé le 30 avril 1852 , mourut en mai 1853 .

54 Nous constatons, par cette lettre, que MM. Bellemare et GérinLajoie fréquentaient la meilleure société du temps, milieu de marchands prospères, d'hommes politiques et de juristes.

55 C'est en 1879 que Dionis Lesieur-Desaulniers, gendre de Raphaël Bellemare, fut nommé traducteur français à la Chambre des Communes et fixait sa résidence, à Ottawa. Peu de temps après le décès d'Antoine GérinLajoie, en 1882, M. Bellemare achetait la maison que celui-ci avait fait cons- 
truire au « Coteau de sable », et qu'il avait habitée avec sa famille, de 1868 à sa mort. M. Bellemare cédait cette propriété à sa fille, Madame D. L.Desaulniers. La famille Desaulniers l'habita jusqu'à ces toutes dernières années. La petite-fille de Raphaël Bellemare, Yvonne Desaulniers, Madame Robert-J. Manion, veuve de l'ancien chef de l'opposition conservatrice, sous le gouvernement King, y mourut en 1951.

56 Ces paroles de M. Bellemare, je les ai entendues rappeler, peu après sa mort, par Mme Desaulniers, elle-même, une tante que j'aimais à visiter souvent.

57 S'il fallait croire le Canadian Biographical Dictionary, nous aurions raison, en effet, de placer l'entrée de $M$. Bellemare, comme secrétaire à la Société St-Jean-Baptiste de Montréal, dès 1848. C'est la date que donne cet ouvrage en 1881. Nous avons tenté de faire confirmer le fait par des pièces originales. Nous nous sommes adressée au Secrétaire actuel de la Société, M. Thomas Bertrand, qui a bien voulu effectuer des recherches. Elles n'ont apporté aucun résultat. Les procès-verbaux, à cette époque, ne portaient point de signature le plus souvent. Nous tenons à remercier $\mathbf{M}$. Bertrand pour son obligeance.

58 Alors l'arrivée du navire français «la Capricieuse en 1855, ne serait plus la première «présence» de la France sur les bords du SaintLaurent. Une autre «présence» spirituelle, et venant de la France catholique doit avoir place dans notre mémoire. La Société de Saint-Vincent-dePaul, fondée au Canada, à Québec, en 1846, recevait de France, des constitutions créées par Ozanam jadis. Toute une correspondance s'engageait entre les directeurs des Conférences de Saint-Vincent-de-Paul de France et du Canada ... Ce sont là des faits, des pièces tangibles aussi. Le mot éloquent de M. Magnan, pourquoi ne pas le justifier avec émotion quand l'occasion s'en présente?

59 Le notaire Ovide Leblanc (1801-1870) est né à Champlain, près des Trois-Rivières, d'une famille d'origine acadienne. Il vint s'installer à Montréal, vers 1842 ou 1843. Il y épousait Sophie Lindsay, veuve de Daniel-H. Saint-Georges-Dupré, député au premier parlement de 1792. Elle décédait en 1852, sans lui laisser d'enfant. Le notaire Leblanc représenta le Comté de Beauharnois au Parlement de 1851 à 1854. Il paraît avoir quitté Montréal vers 1860, pour se fixer à Portage-du-Fort, dans le comté actuel de Pontiac. Ovide Leblanc serait mort en cet endroit. (Voir dans le Bulletin des recherches historiques (Lévis, 1952), 58: no 4, un article très élaboré de Louis Richard, d'où nous avons tiré les quelques détails ci-dessus).

60 Voir les Noces d'or de la Société Saint-Vincent-de-Paul à Québec, 1846-18.96 [Editées par Mgr Têtu], (Québec, Librairie Montmorency-Laval, Pruneau et Kirouac, libraires-éditeurs, 46, rue la Fabrique, 1897), 203-204.

61 Robert Rumilly, de l'Académie canadienne-française, La plus riche aumône - Histoire de la Société Saint-Vincent-de-Paul au Canada (Montréal, éd. de l'Arbre, 1946), 233 pages. $20 \times 13.5 \mathrm{~cm}$.

62 Ibid., 106. M. Rumilly donne comme référence: Rapport du Conseil particulier de Montréal au Conseil supérieur de Québec pour l'année 1900 (Archives de la Société Saint-Vincent-de-Paul, à Québec).

63 Monseigneur de Forbin-Janson (1785-1844) vint au Canada en 1840. Le 21 janvier 1841, l'évêque de Nancy installe à Montréal le Chapitre de la Cathédrale. Il prêche à divers endroits, puis dirige des retraites. Le 6 août, il bénit à Saint-Hilaire la croix de la montagne. Le 26, il préside à Bytown (Ottawa) à la bénédiction de la pierre angulaire de la cathédrale. Le 15 août 1842, Monseigneur remplit une mission diplomatique à Londres et en Irlande en faveur de l'élargissement des Canadiens déportés à la 
Nouvelle-Galles-du-Sud. Il meurt le 11 juillet 1844. Nous n'avons tenu compte que de ses bons offices envers le Canada, car sa vie, close à 59 ans, le fait voir d'une extraordinaire ardeur apostolique. (Voir Allaire, Dictionnaire biographique du clergé canadien-français $(1910), 211$.

64 Edmond-Octave Hardy-Chatillon, né à Québec en 1831, fit ses études au Séminaire de Québec, puis entra chez les Jésuites. Il quitta la Compagnie de Jésus pour cause de santé, fut professeur de musique à Sainte-Thérèse, et enfin au Collège de Nicolet, à partir de 1862. Il y mourut en 1892. (Mgr Douville, op. cit., II, passim).

65 Louis-François Laflèche (1818-1898), le deuxième et célèbre évêque des Trois-Rivières, était né à Sainte-Anne-La-Pérade. Il fit ses études classiques au Collège de Nicolet, $\mathrm{y}$ professa, $\mathrm{y}$ fut même directeur des études et supérieur du Collège. Grand Missionnaire à la Rivière-Rouge avec $\mathbf{M g r}$ Provencher, puis à l'Ile-à-la-Crosse, avec Mgr Taché, il allait être un non moins grand évêque, aux Trois-Rivières, de 1867-1898. Il décédait dans son diocèse le 4 juillet 1898, âgé de 80 ans.

66 Isaac-Stanislas Desaulniers (1811-1868) était originaire d'Yamachiche. Il fut élève au Collège de Nicolet. Frère du savant professeur des sciences mathématiques, de physique, etc. dans cette institution, il allait, pour sa part, enseigner la philosophie au Collège de Saint-Hyacinthe. Il fut l'introducteur au Canada de la doctrine de saint Thomas. Il mourut supérieur du Collège et Grand vicaire de l'évêque de Saint-Hyacinthe, le 5 avril 1868. Le Sénateur Louis-Olivier David a écrit une courte biographie du personnage en 1883. Napoléon Bourassa, peintre et écrivain, a laissé un beau portrait de l'abbé Isaac Desaulniers, conservé au Collège de SaintHyacinthe. Puis-je ajouter qu'il fut un grand-oncle dont on me racontait des traits intéressants durant ma jeunesse?

67 Voir Nos croisés ou Histoire anecdotique de l'expédition des volontaires canadiens à Rome, pour la défense de l'Eglise (Montréal, Fabre et Gravel, lib.-éd., 1871), 27.

68 Ibid., 26.

69 Voir The First Hundred Years. History of the Montreal City and District Savings Bank, 1846-1946, by Taggart Smyth, D.P. Sc [Montreal s.d.] 180 pages. Illustrations, portraits. $21 \times 14.5 \mathrm{~cm}$.

N.B. Nous devons à M. Jacques Bourgeois, publiciste de la Banque d'Epargne (bureau central de la rue iSt-Jacques ouest), le don de la luxueuse plaquette ci-dessus décrite, et plusieurs renseignements qu'il m'a fournis avec beaucoup d'obligeance. Nous l'en remercions vivement. Ajoutons qu'une belle toile présentant le portrait de $\mathrm{M}$. Bellemare décore les murs de la succursale de la Banque d'Epargne, angle des rues Saint-Denis et Rachel.

70 Ibid., 7.

71 Ibid., 33.

72 Ibid., 147.

${ }^{73}$ Voir les Bases de l'Histoire d'Yamachiche, 1703-1903. Ses fiefs, ses seigneuries, ses premiers habitants ... et autres renseignements tirés de manuscrits originaux inédits, conservés dans les vieilles archives du Bas-Canada, par Raphaël Bellemare, journaliste... secrétaire de la Société historique de Montréal, etc. (Montréal, C.-O. Beauchemin \& Fils, librairesimprimeurs, [1904], 18-20).

74 Ibid.

75 Voir Léon Gérin, Antoine Gérin-Lajoie, op. cit. Voir aussi note 52. 
76 Joseph-Ubald Baudry (1816-1876) est un montréalais d'origine. Admis au Barreau en 1838, il allait faire des sciences juridiques, l'étude de toute sa vie. Il fit cependant partie, de 1847 à 1850, du Conseil de la ville de Montréal. En 1850, il devient greffier des Appels, greffier aussi de la Cour seigneuriale. A la demande de Sir Georges-Etienne Cartier, il fut adjoint à M. Ramezay, en 1859 , comme secrétaire de la Commission de la Codification des lois. Quatre ans plus tard, il devenait codificateur de nos lois. Il fut nommé juge de la Cour supérieure pour le district de Montréal en 1869, co-fondateur de la Revue légale et de jurisprudence et aussi de la revue Décisions des Tribunaux du Bas-Canada. L'histoire canadienne l'intéressait profondément. Il fut l'un des co-fondateurs de la Société historique de Montréal. Il décédait à Montréal, le 12 janvier 1876. M. Ubald Baudry, président actuel (1959) de la Société historique de Montréal, est un de ses descendants.

77 Louis-François-Georges Baby (1832-1906) est né à Montréal. Il eut comme ancêtre, au Canada, Jacques Baby de Ranville, sergent au régiment de Carignan-Salières, à son arrivée au Canada en 1665. La belle carrière de Louis-François Baby qui fut avocat, député, ministre, magistrat, chevalier de saint Grégoire-le-Grand, fut souvent narrée. Le Père LeJeune, o.m.i., dans son Dictionnaire général du Canada (ouvrage cité, Ottawa, 1931), I: 110-111, observe qu'« il portait le plus vif intérêt à toutes les recherches historiques, devint l'un des fondateurs de la Société historique de Montréal. $\gg$ Nous ajoutons qu'il en fut le premier secrétaire, de 1857 à 1860 . Il venait d'être admis au Barreau et ne comptait, en 1857, que 25 ans. Plus tard, il devint président de la Société d'archéologie et de numismatique de Montréal. En 1889, le Pape Léon XIII lui conférait le titre de Chevalier de l'Ordre de Saint-Grégoire-le-Grand. En 1880, il avait été nommé juge puîné à la Cour Supérieure; puis, en 1896, président de la Commission de revision des statuts. Il mourut en 1906. Pierre-Georges Roy, dans les Juges de la Province de Québec (Québec, 1933), 27, termine la notice biographique consacrée au juge Baby par le rappel des trésors historiques qu'il a patiemment amassés durant sa vie: "Collectionneur averti, écrit-il, de monnaies et de choses canadiennes,... il possédait à sa mort une des plus riches collections du pays en monnaies, médailles, livres, manuscrits canadiens, etc. qu'il légua à l'Université de Montréal. 》 M. Camille Bertrand, auteur d'une Histoire de Montréal, archiviste et paléographe, a été chargé par l'Université de Montréal, il y a quelques années, de faire l'inventaire des manuscrits, papiers de famille, etc., du juge Baby. Cet inventaire a été publié dans une des livraisons de l'Action Universitaire (avril, 1951), no 3: 27-41.

78 Voir, dans les Cahiers des Dix (Montréal, 1943), no 8: 13-54, le précieux article de $M$. Victor Morin, intitulé «L'odyssée d'une Société historique 》.

\section{Ibid., 54.}

${ }^{80}$ Le moment présent ne se prête-t-il pas à la publication des lettres de M. Bellemare à Mgr Hamel ? L'Archiviste de la Province de Québec, M. Antoine Roy, vient de publier son Rapport pour 1955-1956 et 1956-1957 où il a inséré (33-176) \&l'Inventaire de la Saberdache de Jacques Viger $\$$. Qui peut douter du plaisir éprouvé par tous les historiens à la vue de ce travail important dû̀ à la science archivistique de M. Fernand Ouellet, du bureau des Archives de la Province? L'introduction de M. Ouellet est vraiment un « morceau à lire et à relire ». Il faut l'en féliciter vivement. L'on comprend davantage maintenant que la Société historique de Montréal ait si profondément regretté de ne pouvoir placer au premier rang, parmi ses propres archives, cette cuvre que son fondateur édifia patiemment, 
durant sa vie, année par année, presque jour par jour. Ce «Bénédictin du Canada $\gg$, comme on l'a appelé, était un rude et bien intelligent travailleur. 81 Le Dr Chabot avait épousé la nièce de l'abbé Verreau.

82 M. l'abbé Nazaire Dubois.

83 Voir note 80.

$R E A B O N N E M E N T$ : C'est dès maintenant - on ne l'oubliera pas - qu'on se réabonne à la Revue d'histoire de l'Amérique française. $\$ 5.00$ par année.

INDEX des dix premières années de notre Revue.

Cet Index indispensable aux bibliothèques, aux chercheurs, vol. de 315 pages, est toujours en vente à nos bureaux: 261 rue Bloomfield, Outremont, Qué., Canada. Prix : $\$ 5.00$ l'exemplaire. 